\title{
Churning, power laws, and inequality in a spatial agent-based model of social networks
}

\author{
Jae Beum Cho' ${ }^{1}$ Yuri S. Mansury ${ }^{2}$ - Xinyue Ye $^{3}$
}

Received: 14 June 2016 / Accepted: 27 September 2016 / Published online: 6 October 2016 (C) The Author(s) 2016. This article is published with open access at Springerlink.com

\begin{abstract}
Amidst many previous network models lacking a spatial dimension, this paper proposes a dynamic agent-based model of social network formation that explicitly considers space. We find that varying the dynamics of agent interaction causes the emergence of differential degree distributions as well as nonlinear dynamics in social and spatial inequalities. The scale-free property of degree connectivity vanishes when tie formation dominates tie dissolution, with power laws re-emerging when tie dissolution is of equal strength or stronger than tie formation. Furthermore, we find a nonlinear relationship between network density and agent inequality in social resources. In particular, multiple phase transitions occur where the relationship is positive in one phase but negative in another. This suggests that, contrary to intuition, higher connectivity can have an adverse distributional impact by benefiting the already privileged. Critically, we find a tradeoff between agent inequality and spatial inequality where the geographic concentration of social resources accompanies a more equal distribution of connectivity. Finally, the disadvantage of agents with limited spatial reach is exacerbated as network density increases. Our results thus highlight the importance of distinguishing between social and spatial inequality in policymaking.
\end{abstract}

\footnotetext{
$凶 \quad$ Jae Beum Cho

jc2664@cornell.edu

Yuri S. Mansury

ymansury@iit.edu

Xinyue Ye

xye5@kent.edu

1 Department of City and Regional Planning, Cornell University, Ithaca, NY 14850, USA

2 Department of Social Sciences, Illinois Institute of Technology, Chicago, IL, USA

3 Department of Geography, Kent State University, Kent, OH, USA
} 
JEL Classification C61 $\cdot$ C63 $\cdot \mathrm{R} 11 \cdot \mathrm{R} 12 \cdot \mathrm{P} 11$

\section{Introduction}

Regional science since its inception has focused on socioeconomic phenomena with a spatial dimension (Nijkamp et al. 2014). Social networks in particular are one of the defining issues of our time that have transformed how we think about socioeconomic phenomena. A growing body of empirical work measuring different aspects of social networks has indeed shown that connections matter for a variety of outcomes, such as getting jobs (Lin and Dumin 1986), becoming more successful entrepreneurs (Greve and Salaff 2003), or maintaining high-performing organizations (Borgatti and Cross 2003). But while it is intuitive to many that social interactions should respect Tobler's (1970) "first law of geography," ground-breaking models of networks such as the preferential attachment model of Barabási and Albert (1999), the random graph model of Erdos and Rényi (1960), or the small-world model of Watts and Strogatz (1998) all assume that geographic space has little to no relevance. Casual empiricism, however, suggests that space matters in important ways. Urban dwellers, for example, rely much more for mutual support on local neighbors than on acquaintances in other cities (Gans 1962; Mansury and Shin 2015). This is in line with a Pew Internet study that shows face-to-face contact has remained the dominant means of communication even for core users of online social network sites (Hampton et al. 2009).

Regional science is well positioned to contribute to the literature on spatial networks as it has long recognized the critical role of physical geography in socioeconomic relationships. Cities in particular are manifestations of the dense interactions among residents living in close proximity to one another (Batty 2013). Situating entities in space therefore strengthens the empirical basis and sheds new light on the nature of social networks. Spatially embedded models of networks indeed show the non-trivial impact of geography on network properties (Kosmidis et al. 2008) as well as the importance of geographically concentrated networks (Browning et al. 2004). Empirical studies by both sociologists (McPherson et al. 2001; Wellman 1996) and regional scientists (Cassi and Plunket 2014; Fritsch and Kauffeld-Monz 2010; Ioannides and Topa 2010) confirm the critical role of space, with social distance (e.g. the frequency of contact or the strength of relations) being heavily influenced by geographic propinquity.

An important feature of contemporary social networks is churning brought about by social actors that continuously re-evaluate and alter their links. For example, it has been observed that more than half of social networking users have "unfriended" contacts in their networks - deleting members from their friends list (Madden 2012). This is also evident in online dating networks, where members who start out as strangers eventually enter a committed relationship (Smith and Duggan 2013). Many of these relationships end up being dissolved in the end. From a broader perspective, it has been argued that rising crime and disorder in cities are in large part brought about by the decay of local social ties (Sampson 2004). Others have gone so far as to suggest that the continuous decline in social connections has impoverished our lives and communities (Putnam 2001). 
The churning dynamics are consistent with the view of networks as complex, dynamic, self-organizing systems embedded in space and governed by individual interactions. The present study therefore examines the evolution of spatial networks using agent-based models (ABMs), a class of complex-systems approximations where the abstraction maintains "a close association with real-world agents of interest" (Miller and Page 2009). Regional scientists in particular have used simulation techniques to explore the bottom-up processes that drive the emergence of spatial patterns (Mansury and Gulyás 2007; Torrens 2007, 2010; Xie et al. 2007). In essence, an ABM is a set of agent-specific rule-based algorithms that shape the outcomes for individual agents. The algorithms allow agents to interact directly with one another, and in so doing link individual changes to systems dynamics. ABMs' main advantage for modeling social networks is that they utilize a more realistic bottom-up approach that gives agency to social actors to collectively generate systems behavior.

This paper addresses the following research questions. The first focuses on power law distributions building upon the observation that while many real-life social networks have highly skewed distributions, many others do not. For example, while citation networks and actor networks are both networks of collaboration, the former tend to follow the power law (Barabasi 2002) even though the latter exhibit small-world properties (Watts 1999). The present study queries when a scale-free distribution is sustainable in a spatial network where agents are allowed to re-evaluate their ties, and conversely under what conditions churning destabilizes the power law. Second, we argue that social capital is a socioeconomic resource affected by the decisions to maintain, dissolve, and form new connections. A natural question then is how the unequal distribution of social capital is affected by the differential strength of network churn factors, and under what conditions the disparities can be mitigated.

Our paper contributes to the literature by adding space into an ABM of preferential attachment (PA) with network churn, and by showing how such refinements result in previously unknown emergent properties and network behavior. In contrast to the sole focus on connectivity in the original preferential attachment model, here we consider the refinement proposed by Bianconi and Barabási (2001) allowing agent fitness to also influence the probability to form a new connection. Space then matters in the specification where proximity affects agent fitness, which intertwines with churning to embed the agent selection mechanism in an evolving spatial landscape. Our model is further enriched by the distinction between two types of agents-namely introverts and extroverts - in a community where introverts are limited in their spatial scope of interactions, while extroverts are free to maintain long range connections. This is in line with the theory and empirics that reveal that psychological traits dictate social interactions (Cuperman and Ickes 2009) and economic outcomes (Tversky and Kahneman 1981).

The next section highlights three key concepts, namely PA, power laws, and homophily. Section 3 elaborates on our network model, and Sect. 4 on the implementation of our ABM in simulation analysis. The penultimate Sect. 5 analyzes our simulation results focusing on the degree distributions of agents under different parameter settings, as well as on the individual and spatial inequalities in social capital. The concluding section discusses key policy implications and recommendations. 


\section{Theoretical framework}

\subsection{Preferential attachment (PA)}

The original PA model proposed by Barabási and Albert (1999) ushered in a boom in network studies in the late 1990s. While the model was formalized in their seminal paper, the idea that the rate in which a particular agent acquires links is proportional to the links that the agent already has - thus preferential attachment-had been around long before. Notably, de Solla Price 1965 discussed the concept of cumulative advantage in the context of scientific citations, while sociologists referred to the same phenomena as the Matthew effect (Merton 1968), named after the Gospel of Matthew: "For whoever has will be given more, and they will have an abundance." (Mt, 25:29). This "rich get richer" mechanism has been observed in many types of networks, ranging from the internet and power grids to social networks (see Barabasi 2002 for examples).

The model assumes a network that starts with a small number of nodes that are randomly connected. In every succeeding step, a new node is added, linking itself to the incumbent nodes already in the network. PA is incorporated by the simple rule that incoming nodes prefer incumbents that are already well connected, thus awarding an incumbent with significant connectivity a higher probability of attracting newcomers. The PA model serves as a good starting point for analyses of social networks due to two key characteristics. First, it features a network that expands continuously through agent and tie addition. Second, new agents connect to others already in the network through a process of selection, which favors more connected agents. The first characteristic is essential in modeling growing networks that are fundamentally different from static networks with a fixed number of nodes and edges (Erdos and Rényi 1960; Watts and Strogatz 1998). The second characteristic captures the fact that in many social networks, agents choose to associate with others that are already well connected. In theoretical terms, selection is introduced by allowing the probability that a node attracts others to be proportional to its degree connectivity, ${ }^{1}$ which is in contrast to random network models in which the probabilities of attachment are fixed in time.

The PA model, however, abstracts away from certain important aspects of real networks. The lack of a spatial dimension in particular is one critical omission to which we devote Sect. 2.3 below. The PA model also ignores network churn, unlike random graph or small-world models that allow for the "rewiring" of links. Thus once an agent is connected to an incumbent upon entry, it ceases to seek new connections and only passively receives links from subsequent newcomers. Ignoring churn, however, disregards the body of empirical literature confirming that social actors constantly re-evaluate network ties based on individual decisions and preferences (Karnstedt et al. 2010; Sasovova et al. 2010). Furthermore, without tie formation and detachment among incumbents, the PA model gives near absolute agency to older nodes, ${ }^{2}$ which is simply not the case in many settings. The present study responds to the challenge

\footnotetext{
1 The degree of a node refers to the number of connections that the node has.

2 This is simply because nodes that have been present for longer periods of time have more opportunities to form links with incoming nodes (see Adamic and Huberman 2000 for a critique).
} 
of developing a spatial agent-based model to study the evolution of networks in a churning environment where relationships ebb and flow.

\subsection{Power laws}

The PA model was conceived to explain the World Wide Web in which a few influential Web sites have a very large number of links, while the rest harbor only a few connections. This highly unequal distribution exhibits a power law, which refers to the linear relationship in the log-log plot of the degree distribution. ${ }^{3}$ Power law distributions are scale-free, a notion that is best understood vis-à-vis the scale-dependent counterparts. Magnitudes such as the length of a town block, the height of a building and the number of bedrooms in a housing unit have a characteristic scale, which means the mean value is representative of the magnitude that one actually observes on the ground. By contrast, power law distributed urban social networks lack characteristic scale, and this means that the average number of acquaintances is not a good predictor of the extent to which a city resident is connected. In stochastic terms, the degree distribution exhibits "fat tails," which implies scale independence, or scale-free.

Urban and regional scholars have long been familiar with regularities in the size distribution of cities (Zipf 1949). More relevant for our study here is the presence of power law relationships in spatial networks, such as those for commuters (De Montis et al. 2009). The essence of power law distributions is captured by the existence of a few nodes with very large degrees, acting as "hubs." Fat-tailed distributions therefore imply wide variations in the extent of social contacts-and thus the resources (Granovetter 2005; Lin 2001)-that a node can tap into. While most have modest connections, a few exert enormous influence by virtue of their hundreds or even thousands of contacts. The implied distribution of resources is therefore highly skewed.

Understanding the sources of inequality in space is one of the central challenges of the science of regions. But before we can begin addressing spatial inequality, we must first disentangle the processes that give rise to differential degree distributions. Under what conditions do networks sustain fat-tailed distributions, and under what conditions does the power law break down? It is important to note that many networks are scale dependent. In the small-world networks that Watts and Strogatz (1998) consider for example, the degree distribution follows a random distribution, and the authors give numerous examples of such networks. Thus while the power law distribution is observed for specific networks, there are many networks that exhibit characteristic scale with small deviations from the average connectivity.

The present study addresses this and other questions, including whether the power law can be sustained in networks where the arrival of new nodes and links occurs at the same time as the elimination of ties among existing nodes. The evidence drawn from our ABM simulations hints at the potential tug of war between tie formation and tie dissolution. The power law seems to persist when dissolution dominates formation, but vanishes in the opposite case.

\footnotetext{
3 The degree distribution of a network describes the relative frequencies of nodes with different degrees (Jackson 2008).
} 


\subsection{Homophily, propinquity, and social capital}

As elegant as the preferential attachment (PA) model may be, it leaves out a number of important variables. Social ties depend not only on connectivity but also on other social and economic indicators. In particular, the model abstracts away from homophily, which refers to the tendency for people to associate with those sharing a wide range of similar attributes. Homophily is one of the most fundamental forces identified to date that have been known to shape social networks (McPherson et al. 2001). Numerous studies of social relationships indicate the dominance of homophily in social interactions, ranging from ties of marriage (Kalmijn 1998) or friendship (Aral et al. 2009; Verbrugge 1983), to membership in voluntary associations (Cornwell and Dokshin 2014) or appearing with others in a public space (Mayhew et al. 1995). The PA model is in essence a model of heterophily, whereby nodes prefer to connect to others that are as different as possible (in the number of links) to themselves. Within the social networks literature, whether it is homophilous or heterophilous ties that predominate has long been debated. Developments in theory and empirics suggest that both are instrumental in explaining social interactions, with one dominating the other under different circumstances (Burt 2005; Mehra et al. 1998).

An important source of homophily is propinquity, as we are more likely to sustain relationships with others that are geographically closer to us rather than with others farther away (McPherson et al. 2001). Zipf (1949) states that the importance of propinquity stems from the notion of effort, for it takes more energy and effort to maintain relationships with distant contacts. Many examples, such as studies of neighborhoods (Campbell 1990), residential proximity (Verbrugge 1983), or immigrant enclaves (Wilson and Portes 1980) show that more homophilous interactions take place when social actors are closer to each other. ${ }^{4}$ Locations thus determine not only physical factors, but also the common traits that forge neighborhoods. It is to account for homophily that we give each node a unique location in the network of heterophilous interactions. The introduction of space allows us to set the probability for tie creation to be inversely related to the relative distance between nodes.

Spatial embeddedness changes the dynamics of tie formation as locations introduce an important new source of heterogeneity. In the preferential attachment model, heterogeneity stems only from differences in connectivity. The introduction of space means that nodes with the same connectivity are not necessarily equally attractive, as it depends on where they are located in relation to the evaluating node. As we will show below, the introduction of space results in degree distributions that generally lack power law properties. While hubs with the highest number of connections are still the biggest draw, we do not expect hubs to be secluded in space as isolation would have prevented them from becoming a hub to begin with.

Closely aligned with the notions of homophily and heterophily is the concept of social capital. It has been theorized that strong homophilous relationships promote higher levels of trust, reciprocity, and enforcement of norms (Coleman 1988). By contrast, weak heterophilous relationships matter when people need to "get ahead,"

\footnotetext{
4 Proximity and preferences come together in Schelling's famous work where people sharing similar traits end up in the proximity of each other despite only mild preference for neighbors who are like them.
} 
with individuals maintaining more such relationships gaining brokerage capacity or better access to non-redundant information (Burt 1992, 2005; Granovetter 1973). Social capital is thus a resource that one can tap into, but unlike physical or human capital, it is embedded in the network fabric that one is a part of (Portes 1998). As we will show below, this conception allows us to examine the inequalities in social capital emerging from the differential propensities to form and dissolve ties.

\section{The model}

In the following presentation of the model, we will use the term agents and nodes interchangeably. We consider a network comprised a set of nodes $N=\{1,2, \ldots, n\}$ and a set of undirected links $L \subseteq N \times N$. Multiple links between two given nodes as well as self-links are assumed to be absent. The network is initially conceived with two nodes that are connected to each other at $t=0$. In each subsequent period, a new node joins the network and must choose which of the preexisting nodes it will connect to. As in the preferential attachment model, a preexisting node is chosen with probability that is proportional to its degree connectivity (Vega-Redondo 2007) as well as its individual fitness. The probabilistic approach is used to represent sources of variability in network formation that are too complex to capture mechanistically. In real networks, ties are not always formed based on connections because of either bounded rationality or factors other than connectivity.

The novel attachment mechanism here stems from the preferential bias for preexisting nodes with higher fitness, captured through the fitness parameter $\eta$, which represents individual-specific characteristics such as social affinity, wealth, or human capital (Bianconi and Barabási 2001). Formally, the probability $\Pi_{i, j}$ that a new node $i$ will connect to an incumbent node $j$ depends on both the connectivity $k_{j}$ and the fitness parameter $\eta_{j}$ such that:

$$
\Pi_{i, j}=\frac{\eta_{j} k_{j}}{\sum_{j} \eta_{j} k_{j}} .
$$

Equation (1) has a straightforward interpretation. Other things equal, a higher connectivity raises the likelihood of being linked to an incoming node, but a lower fitness would lower this probability. A node searching for a new contact evaluates an existing node $j$ 's fitness based on the combination of two factors, namely human capital $\lambda_{j}$ and the distance between the two nodes $d_{i j}$, such that:

$$
\eta_{j}=\frac{\lambda_{j}}{d_{i j}^{2}} .
$$

The level of human capital $\lambda_{j}$ is drawn from a uniform random distribution with support $[0,1]$. Note that Eq. (2) introduces space by assigning every agent a unique location in the network so that no two nodes can occupy the same area.

A central feature of the tie dynamics in our model is the formation and dissolution of ties among incumbent nodes. The trajectory is cumulatively driven by a combination of tie creation and tie deletion, leading to network churning (Koka et al. 2006). For 
tie formation, we introduce the notion of maximum visibility reach that distinguishes extroverts from introverts. The former are capable of connecting to other agents anywhere within the spatial grid, while the latter are agents that are unable to connect to other agents outside of their visibility range $(v)$. Formally, the probability $\Pi_{e, j}^{F}$ for an extrovert $e$ to connect to any other node $j$ that is not yet a link neighbor is calculated as:

$$
\Pi_{e, j}^{F}=\theta_{f} \frac{\eta_{j} k_{j}}{\sum_{j \notin S_{e}} \eta_{j} k_{j}}
$$

where $\theta_{f}$ is the tie formation parameter drawn from the interval $[0,1]$, and $S_{e}$ is the set of node $e$ 's link neighbors. By contrast, the tie formation probability for introverts $i$ is defined as:

$$
\Pi_{i, j}^{F}= \begin{cases}\theta_{f} \frac{\eta_{j} k_{j}}{\sum_{j \notin S_{i}, d_{i j} \leq v} \eta_{j} k_{j}} & \text { when } d_{i j} \leq v \\ 0 & \text { otherwise. }\end{cases}
$$

Equation (4) assigns a nonzero probability for an introvert $i$ to connect to agents within $i$ 's visibility range, $d_{i j} \leq v$, or zero otherwise.

For tie decay, the probability that any node $i$ deletes a tie with a current link neighbor is determined by the number of links currently maintained by that agent $\left(k_{i}\right)$ and the distance between the two nodes $\left(d_{i j}\right)$. This is based on the notion that maintaining ties requires social effort (Lin 2001), and hence other things equal, nodes with a larger number of links lose connectivity at a higher rate compared to nodes with only a few links. At the same time, links between nodes farther away have a higher chance to dissolve. Formally, we introduce a tie decay parameter that controls how ephemeral ties are within the network. The equation that governs the probability for node $i$ to dissolve its link to neighbor $j$ is as follows:

$$
\Pi_{i, j}^{D}= \begin{cases}\theta_{d} k_{i} \frac{d_{i j}^{2}}{\sum_{j \in S_{i}} d_{i j}^{2}} & \text { when } \theta_{d} k_{i} d_{i j}^{2}<\sum_{j \in S_{i}} d_{i j}^{2} \\ 1 & \text { otherwise }\end{cases}
$$

where $\theta_{d}$ is the tie dissolution parameter drawn from the interval $[0,1] .^{5}$

To capture individual network resources, we utilize the concept of social capital, defined as the instrumental resources that are available to actors through the social ties they maintain (Lin 2001). Formally, we utilize a simple definition of an agent's individual social capital $S C_{i}$ as the sum of the human capital (Coleman 1988; Moretti 2004) of an agent's first-degree link neighbors such that:

$$
S C_{i}=\sum_{j \in S_{i}} \lambda_{j}
$$

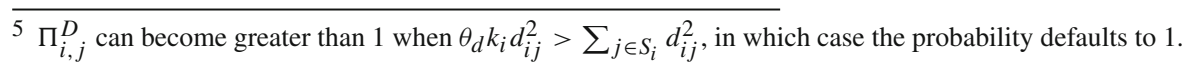


and naturally we define aggregate social capital in the network to be $\sum_{i} S C_{i}$. It is important to note that social capital — both at the individual and aggregate levelsindirectly feeds back into our model in the form of influencing individual decisions of tie formation and dissolution. From the setup, the individual social capital measure depends on both the number of links (which increases the number of link neighbors $j$ that are included in set $S_{i}$ ) as well as the quality of these links (the human capital that a link neighbor is endowed with). Agents with more links will, ceteris paribus, have more social capital, which feeds back to them by (1) increasing their chances of being linked by other agents, while at the same time (2) increasing their likelihood of losing links to others due to higher values of $k_{i}$ which increases $\Pi_{i, j}^{D}$ [see Eq. (5)]. On the other hand, agents with higher quality links, ceteris paribus, will be affected by an increased chance of losing links, for their link neighbors will usually harbor a larger number of connections which would increase the chance of tie dissolution. Aggregate social capital also feeds back to individual agents, with higher levels resulting in lower tie formation due to network saturation (i.e., less possible links to be formed) as well as higher tie dissolution (i.e., more possible links to be severed).

Note that there are two mechanisms that act against each other to determine levels of social capital; namely (1) the mechanism of preferential attachment based on degree, human capital, and distance, and (2) the mechanism of preferential detachment based on degree and distance. We will assess how individual and aggregate levels of social capital — as well as inequality in social capital—evolve for varying values of the tie formation $\left(\theta_{f}\right)$ and tie decay $\left(\theta_{d}\right)$ parameters.

\section{Algorithm implementation}

We use the standard protocol of "Overview, Design concepts, and Details" (ODD, see Railsback and Grimm 2011) to describe the agent-based algorithm. ODD provides a standardized way of presenting the ABM starting with three elements which overview the model and how it is designed, followed by specific design concepts that illustrate the ABM's key characteristics, and ending with three elements that describe the initialization and implementation details. We highlight the main purpose of the model, as well as the entities, variables, scale, and processes below (see "Appendix 4" for specific details of the model according to the ODD protocol).

\subsection{Purpose}

The model seeks to examine how churning affects network resources and system characteristics in a spatial setting. Specifically, we consider how the degree distribution of agents differs based on varying strengths of tie formation and dissolution, as well as how these factors influence individual and spatial inequalities.

\subsection{Entities, state variables, and scales}

The model has three types of entities: individual agents, their network connections, and square patches of residential locations. The network connections are assumed 
to be binary (i.e., either a link exists or it does not), non-redundant (i.e., no more than one link can exist between any two agents), undirected (i.e., the direction of the link is indistinguishable), and containing no self-loops (i.e., no links to oneself). Each patch is in the state of either empty or occupied by maximum one agent, and make up a square grid landscape of $100 \times 100(L=100) .{ }^{6}$ Since opposite edges are disconnected, the landscape represents a two-dimensional Euclidean surface. This is to facilitate the analysis of spatial inequalities between agents as well as specific core-periphery structures that are better represented in two-dimensional space. The patches have no state variables other than their relative position within the grid, which dictates the distances to other patches, and thus the distances between agents.

Agents seek to expand the extent of their connections in the endeavor to increase their social capital while being constrained by the effort required to maintain their ties. Each individual agent is defined by both static and dynamic state variables. The static state variables comprise the agent type (whether extrovert or introvert), level of human capital, the propensity to form ties among incumbents (the tie formation parameter) and to dissolve existing ties (the tie dissolution parameter), and spatial coordinates, which are all predetermined at agent birth. The dynamic state variables are the individual degree connectivity, relative fitness, and social capital. The global variables are the degree distribution, aggregate social capital, and measure of inequality (the Gini coefficient). The model's temporal scale is such that each simulation is run until the population of agents $(N)$ reaches 1000 , which results in a maximum link count of 499,500.

\subsection{Process overview and scheduling}

There are three processes in the model: (1) the linking of a newcomer to an incumbent member of the network, and the (2) formation and (3) dissolution of ties among incumbents. Regarding the first process, in each period a new agent enters the system and is assigned a random location on the spatial landscape and with equal probabilities is deemed either an introvert or extrovert. Introverts are assigned a visibility range parameter value $(v)$ of $15 .^{7}$ After each incumbent node is assigned a value of $\Pi_{i, j}$ [see Eq. (1)], the newcomer first chooses an incumbent at random and creates a link with probability $\Pi_{i, j}$. In the event that the newcomer fails to create a link, it would continue to draw randomly from the full set of incumbents until a connection is formed. ${ }^{8}$ Tie formation between incumbents and tie dissolution are implemented using an exhaustive search method where each incumbent evaluates the probabilities for tie formation [see Eqs. (3) and (4)] and tie decay [see Eq. (5)] for all candidate agents (i.e., all agents that are not currently linked for tie formation, and all agents that are linked for tie decay) in every time step, forming and deleting links accordingly. The sequence is such that first a newcomer is added to the network and linked, followed

\footnotetext{
6 Sensitivity analysis is run for different world sizes. See "Appendix 3".

7 As with world size, sensitivity analysis is run for differing visibility ranges. See "Appendix 3."

8 In other words, the re-selection is done with replacement.
} 


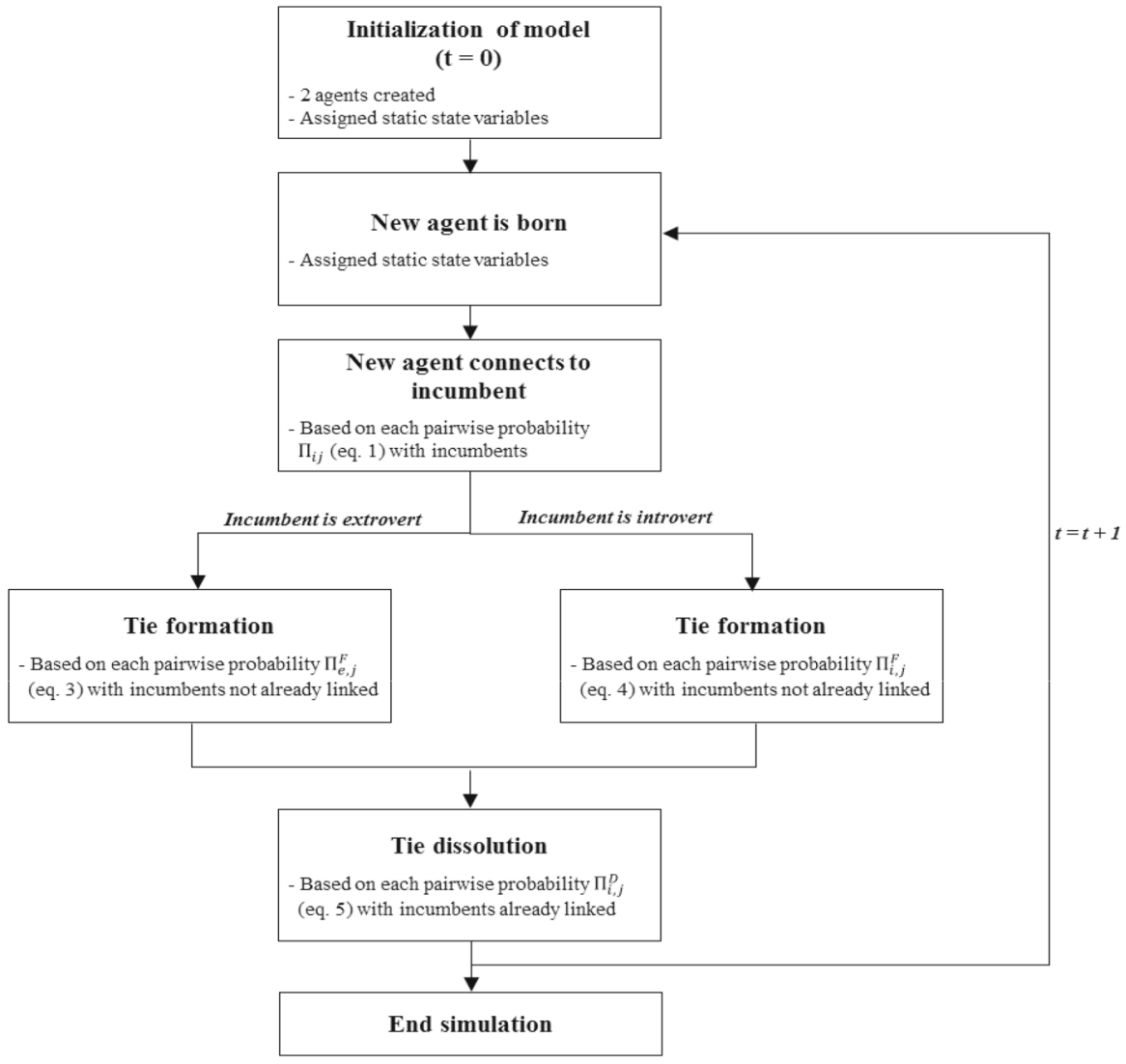

Fig. 1 ABM flowchart

by tie formation and afterward tie deletion processes for the incumbents. ${ }^{9}$ One time period ends when all three processes have been completed for all agents (Fig. 1).

We conduct a parameter sweep on the tie formation parameter $\theta_{f}$ and the tie dissolution parameter $\theta_{d}$, each of which is allowed to vary between 0 and 1 . The analyses employ the mean of five simulation runs for each parameter setting with a unique random number seed for every configuration. ${ }^{10}$ All simulations are implemented in NetLogo (Wilensky 1999), a multi-agent programmable modeling environment popularly used worldwide. We examine the implications of different parameter settings for degree connectivity, network churning, and the presence or absence of power law

\footnotetext{
9 Thus it is possible to delete a link with an incumbent for which an agent has created a link in the same time period.

10 We choose five simulations as the optimal level that balances computational burden with potential variability, as our results suggest that the overall variability of network characteristics is minimal across different random seeds. This is due to the fact that each agent is subject to a stochastic process of initial linkage, link formation, and decay with every other agent in the network at every time period. The enormous amount of stochasticity involved with constructing a final network of 1000 agents thus renders the resulting network as a whole robust to large variations in aggregate characteristics.
} 
distributions. We also examine the distributional impact (measured by the Gini coefficient) of these settings, calculated by assessing the level of social capital obtained by the agents through their network ties. Finally, we consider the spatial distribution of agents with high or low levels of social capital to examine the role of network churn in a spatial context.

\section{Simulation results}

\subsection{Degree distributions and the power law}

In seeking to establish the conditions in which the power law prevails, we first verify our agent-based algorithm by confirming that the power law holds when $\theta_{f}=\theta_{d}=0$, i.e., for the original preferential model of Barabási and Albert (1999) in which incumbents are not allowed to form or dissolve ties with one another. Figure 2a shows that indeed the power law prevails in the long-run distribution of degree connectivity with this parameter setting, with a coefficient of determination $R$-squared of 0.9 and a power exponent of 1.9 which, as it turns out, roughly coincides with the lower bound of the range for many real networks (Barabási and Albert 1999).

The scale-free property, however, vanishes as soon as a small but positive propensity to form new ties is introduced among incumbent agents while holding $\theta_{d}=0$. Figure $2 b$, c reveals that for networks where links never decay, a slight increase in $\theta_{f}$ results in the complete breakdown of the power law. The shifting of the points on the plot to the right as $\theta_{f}$ increases shows how rising propensity to form new ties among incumbents benefits the relatively disadvantaged (in the number of links) by awarding them with more connections.

The power law returns when tie formation and decay are both present, but only when they are either roughly equal in strength or when the rate of tie dissolution is greater than that for tie formation. Figure $2 \mathrm{~d}$ shows the resurgence of the scale-free property when $\theta_{f}=0.4$ and $\theta_{d}=0.5$, accompanied by a highly unequal distribution of social capital as evident from the Gini coefficient that is at least twice that for cases where churning is absent (i.e., when $\theta_{f}=\theta_{d}=0$ ). This suggests that power law distributions that seem ostensibly similar could have vastly different implications for social equity. Churning characterizes most real-world networks, and our model predicts a much higher concentration of social capital at the top than the canonical PA model when both tie formation and decay are present. However, the power law breaks down again when incumbents form ties at a rate that far exceeds the rate at which ties are dissolved.

Figure $2 \mathrm{e}-\mathrm{h}$ shows that the degree distribution approaches a bell-shaped curve as the ratio $\theta_{f} / \theta_{d}$ increases. At the same time, social capital becomes more evenly distributed as the Gini coefficient declines.

Our results also reveal that the power law prevails only in networks with very low density. ${ }^{11}$ Under the original PA model, a network grown starting from two agents

\footnotetext{
11 Network density is simply the number of links $K$ divided by the number of possible links $\hat{K}$, where $\hat{K}=n(n-1) / 2$ and $n$ is the number of nodes in an un-directed network.
} 

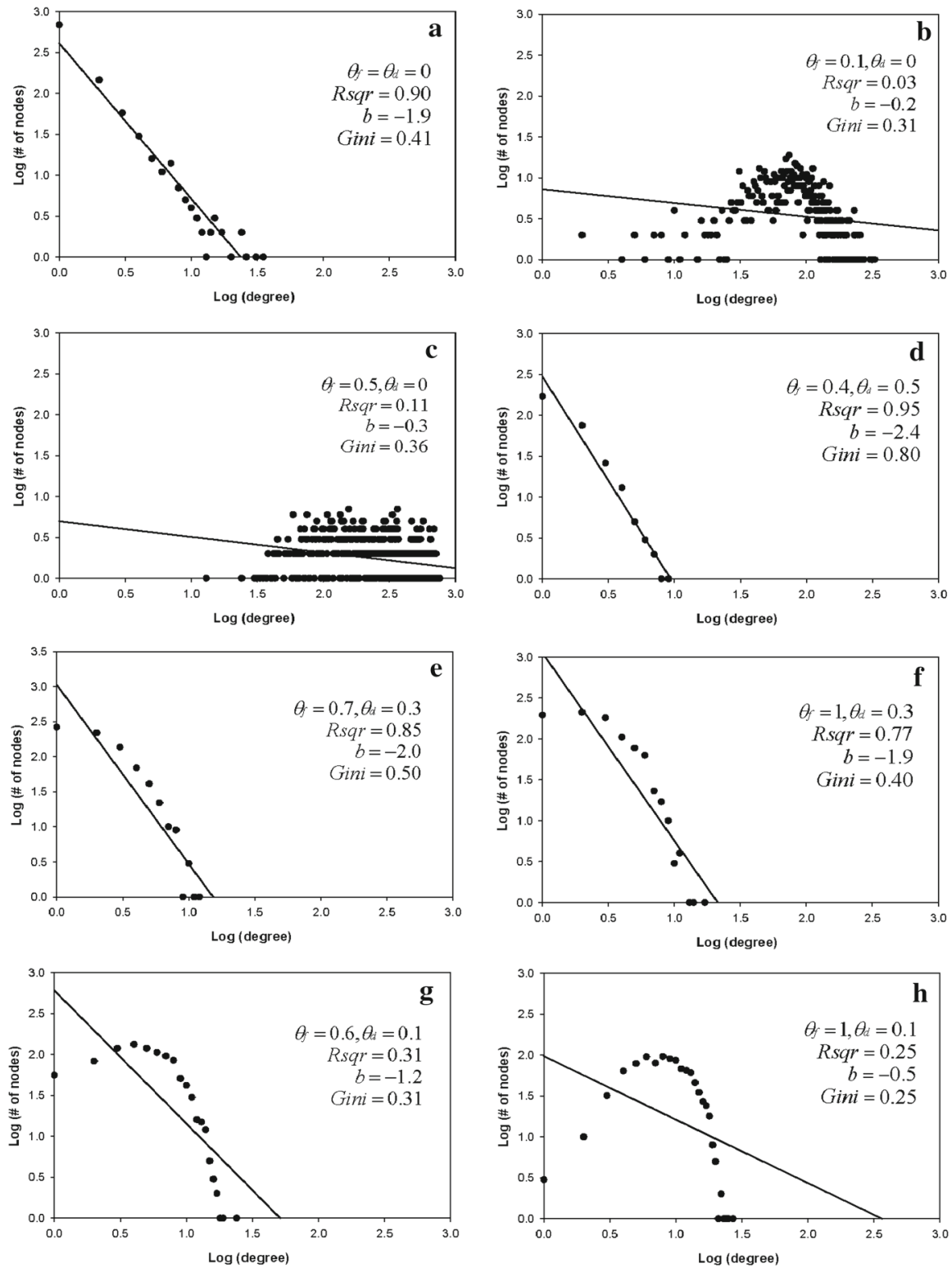

Fig. 2 Degree distributions for select parameter settings. The $X$-axis corresponds to the degree of nodes, while the $Y$-axis to the number of nodes with such degrees, in $\log -\log$ scale

up to 1000 agents in increments of 1 inevitably results in a link count of 999, which corresponds to a network density of $0.2 \%$. The power law network depicted in Fig. $2 \mathrm{~d}$ has a link count of 236 and a much lower density of $0.047 \%$. As we have shown, when tie formation dominates tie dissolution-resulting in a larger number of links and higher density — the power law breaks down. 


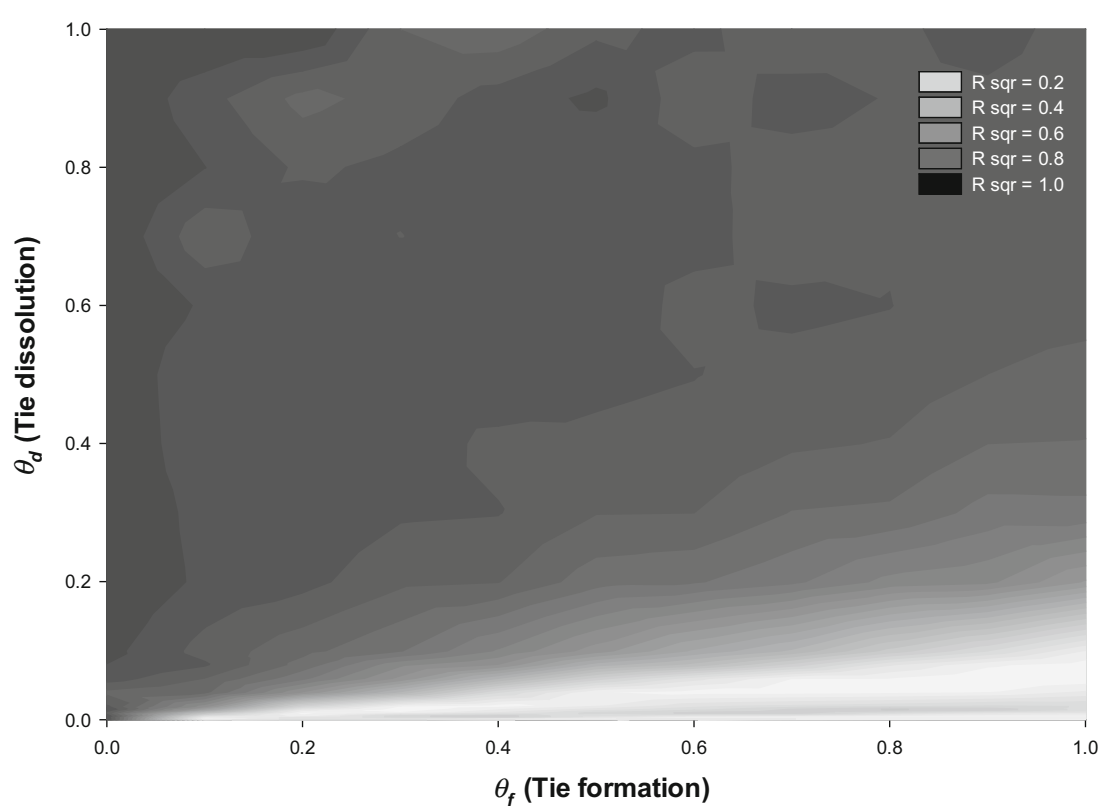

Fig. 3 Relationship between power law fit ( $R$-squared) and network churn parameters. Darker colors represent higher $R$-squared. Values are the mean over five simulation runs each with a different random seed for every parameter configuration

Figure 3 depicts the fit of the power law curves under different combinations of the churn parameters $\theta_{f}$ (tie formation) and $\theta_{d}$ (tie dissolution). Our results suggest that in general, higher values of $\theta_{d}$ relative to $\theta_{f}$ result in distributions that more closely follow power laws. The upper left portion of the figure is dominated by distributions with $R$-squared greater than 0.8 . There is, however, substantial variation in the upper left extremes due to the sparseness of the network in these extremes as well as due to the stochastic evolution of the degree distributions. The figure confirms the results from Fig. 2, with the power law fit decreasing substantially as tie formation becomes more prominent. In addition, it can be seen that introducing tie formation has a much larger effect in breaking the power law for lower values of $\theta_{d}$. For example, when $\theta_{d}=0.6$, an increase of $\theta_{f}$ from 0 to 0.5 still results in a degree distribution with $R$-squared of roughly 0.9 . However, when $\theta_{d}=0.1$, similar $R$-squared values can be obtained only when $\theta_{f}$ is less than 0.1 .

The ability of ABMs to capture system dynamics is advantageous in studying the evolution of networks. Under two different parameter configurations that both result in power law distributions, we show how the same system properties may emerge albeit with different micro-foundations. Figure 4 depicts network formation for when (1) $\theta_{f}=\theta_{d}=0$ (Fig. 4a-d), and when (2) $\theta_{f}=0.4, \theta_{d}=0.5$ (Fig. 4e-h), both of which exhibit power law properties (see Fig. 2). In the absence of churning, the evolution of degree connectivity is path dependent, much like in the canonical PA model that awards higher degrees to incumbents that have been in the network longer. Thus the nodes in Fig. 4a that initially command relatively higher connectivity in 

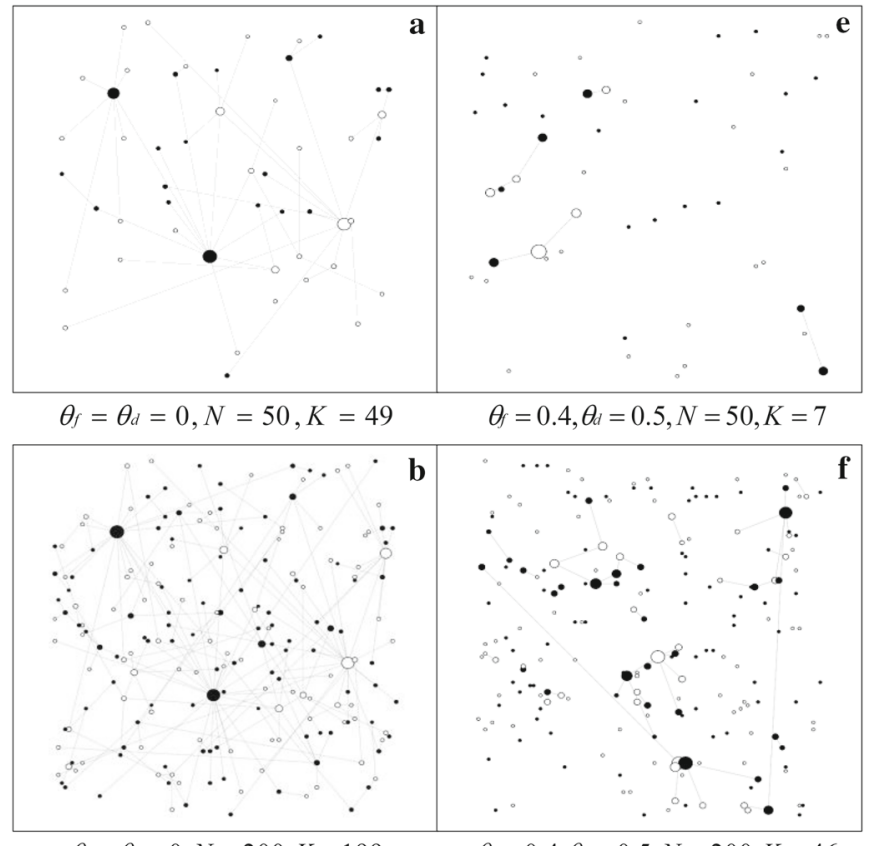

$\theta_{f}=\theta_{d}=0, N=200, K=199 \quad \theta_{f}=0.4, \theta_{d}=0.5, N=200, K=46$

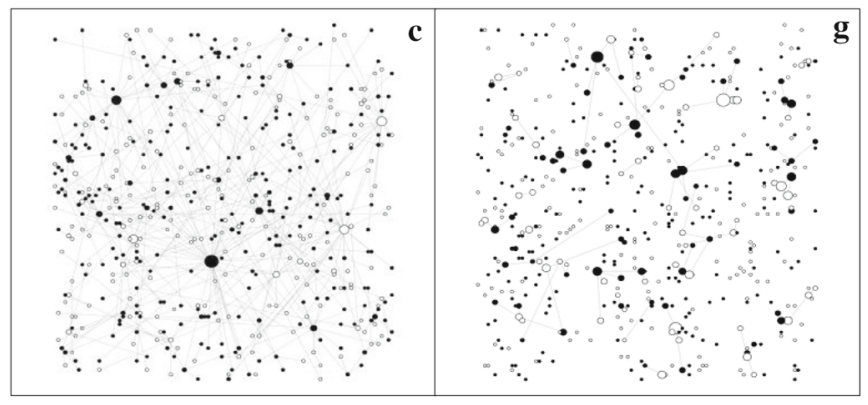

$\theta_{j}=\theta_{d}=0, N=500, K=499 \quad \theta_{j}=0.4, \theta_{d}=0.5, N=500, K=106$

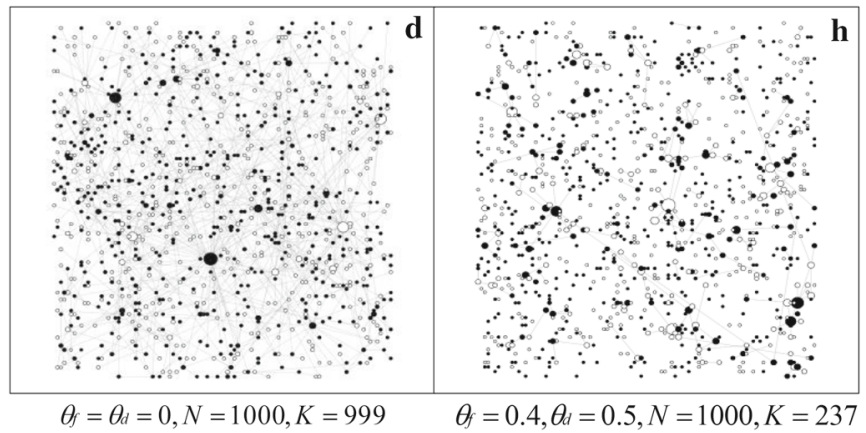

Fig. 4 Network formation dynamics under two different parameter configurations. $\mathbf{a}-\mathbf{d} \theta_{f}=\theta_{d}=0$ while e-h $\theta_{f}=0.4$ and $\theta_{d}=0.5$. Black and white nodes are extroverts and introverts, respectively. Nodes are sized proportionately to their degree 
the early stages are able to maintain their advantage throughout. However, when network churn is present, those that initially had greater connectivity (Fig. 4e) quickly lose their advantage, being replaced by other nodes that were able to get ahead at different times. Thus while overall the two systems both converge to power law distributions, the trajectories are vastly different. This suggests that the original PA model is a special case within the general class of models for which the power law prevails. Furthermore, our results reveal that distinct bottom-up dynamics likely lead to differential access to social capital (see Fig. 4) even for systems that exhibit similar macroproperties.

\subsection{Aggregate social capital}

Figure 5 and "Appendix 1" display long-run levels of aggregate social capital and total link count $K$ for different values of $\theta_{f}$ and $\theta_{d}$. As Fig. 5 shows, aggregate social capital falls monotonically with higher propensity $\left(\theta_{d}\right)$ for incumbents to dissolve ties while holding tie formation $\left(\theta_{f}\right)$ constant. Such results are intuitively appealing, in that we should expect to see lower levels of trust and therefore social capital in a fluid environment where ties are easily broken. Furthermore, the marginal impact of higher values of $\theta_{d}$ tends to diminish for larger values of $\theta_{f}$ (see also "Appendix 1," Table 1)). For example, an increase in $\theta_{d}$ from 0.5 to 0.6 while holding $\theta_{f}$ fixed at 0.5 results in a $30 \%$ decline in aggregate social capital, but when $\theta_{f}$ is fixed at 1.0, the same increase in $\theta_{d}$ results in a decrease of only $20 \%$. This suggests that, other things equal, greater inclination to form new ties increases the resiliency of social capital to forces that dissolve existing ties.

Conversely, Fig. 5 also shows that a higher inclination $\left(\theta_{f}\right)$ to connect to other incumbents is the rising tide that lifts aggregate social capital across different values

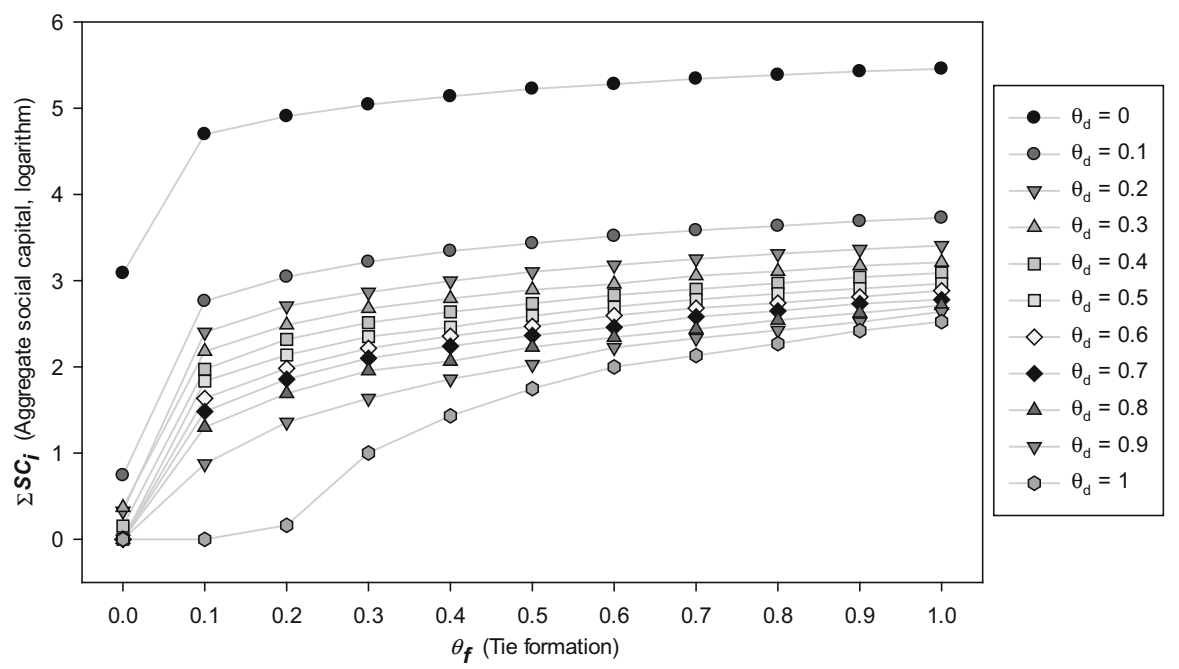

Fig. 5 Tie formation $\left(\theta_{f}\right)$, decay $\left(\theta_{d}\right)$, and aggregate social capital. Values are the mean over five simulation runs each with a different random seed for every parameter configuration 
of $\theta_{d}$. This can be traced to aggregate social capital being proportional to the total number of links in the network, which increases as $\theta_{f}$ rises. Less obvious is the finding that the marginal effect of tie formation diminishes at higher $\theta_{f}$ values. For example, a rise in $\theta_{f}$ from 0.4 to 0.5 holding $\theta_{d}$ constant at 0.5 brings about a $35 \%$ increase in total social capital (see "Appendix 1"), but a rise in $\theta_{f}$ from 0.9 to 1.0 again at $\theta_{d}=0.5$ results in a much lower increase of $14 \%$. In hindsight, this is internally consistent with the logic of the model. Everybody is connected to almost everybody else when $\theta_{f}$ is already near maximum, and so a further increase in $\theta_{f}$ will only have a limited effect on total social capital.

Unexpectedly, however, the marginal impact of tie formation accelerates for larger $\theta_{d}$ 's. Using part of the above example, an increase in $\theta_{f}$ from 0.4 to 0.5 at $\theta_{d}=0.5$ brings about a $35 \%$ increase in total social capital, but the same increase at $\theta_{d}=1.0$ triples the increase to over $107 \%$. It appears that while rapid decay (high $\theta_{d}$ ) foments a sparse network, it is precisely this limited connectivity that allows a higher rate of tie formation to exert greater influence on aggregate social capital. This can be explained by the higher chances to connect to agents endowed with higher human capital when overall connectivity is low.

An important emergent outcome is the nonlinear relationship between network churn and aggregate social capital. Figure 5 reveals a drastic decrease in social capital by a factor of 58 and a decrease in the number of connections by a factor of 62 (see also "Appendix 1") when $\theta_{d}$ is raised from 0.0 to 0.1 . This is in contrast to changes at higher $\theta_{d}$ values where on average the decrease is less than twofold for every 0.1 increment in $\theta_{d}$. These results suggest a phase transition — defined as a significant change of state when parameter values cross a certain threshold (Solé et al. 1996)_occurring in the vicinity of $\theta_{d}=0$, where the qualitative behavior of the system undergoes a significant alteration. Considered a signature of complex networks (Castellano et al. 2000; Holme and Newman 2006), in our case the phase transition is the change from a very sparse network with an average network density of $0.12 \%$ for nonzero values of $\theta_{d}$ to a relatively dense network with an average density of $29.4 \%$ when $\theta_{d}=0$.

The simulation results thus highlight the important role of tie dissolution when analyzing different types of networks. Our findings suggest that networks in which ties are permanent are distinct from those in which ties are transient. Networks with permanent ties have significantly greater connectivity as the high density suggests, which results in higher levels of aggregate social capital. The introduction of even a small likelihood for ties to dissolve, however, shifts the system into one in which connectivity is much lower, and this transition occurs suddenly rather than gradually. The importance of such a distinction has been empirically observed. For example, Wilson and Portes (1980) document the experiences of immigrant minorities integrating into the US labor market, with findings suggesting that immigrant groups in enclaves characterized by more permanent ethnic and cultural ties frequently perform better economically than other minorities that are more fragmented spatially (and thus part of more transient social networks). The phase transition in our model appears in line with such evidence. 


\subsection{Inequality}

Figure 6 and "Appendix 2" show the implications of network churn for the distribution of social capital measured by the Gini coefficient. Figure 6 a reveals that tie dissolution and the Gini coefficient are in general positively correlated, suggesting that a higher rate of tie decay amplifies inequality. This is an unexpected, emergent outcome because ties dissolve more rapidly for highly connected agents [see Eq. (5)]. Since agents with higher human capital also maintain more links, tie decay is expected to equalize the distribution of social capital. But while indeed higher rate of tie decay causes high human capital agents to lose links faster, the preferential attachment (PA) mechanism ensures that the majority of these links are to lower human capital agents. As it turns out, agents with lower human capital lose ground in relative terms, and inequality rises as a result.

On the other hand, a higher rate of tie formation holding $\theta_{d}$ constant generally reduces inequality, unless ties are relatively permanent. Here it is important to recognize the two opposing forces at work. The first is the mechanism of preferential attachment (PA) initiated by the agent itself (or active PA). For an agent endowed with relatively low human capital, a new tie to a high human capital agent increases the former's social capital more than the latter's, and active PA thus tends to reduce inequality. The second is through being preferentially attached to (or passive PA) by other agents within the network. Passive PA favors the well-endowed, and thus tends to accentuate the gap between the highly connected and the relatively isolated. It turns out that which one dominates depends on the magnitude of the decay parameter $\theta_{d}$. When ties are permanent $\left(\theta_{d}=0\right)$, the effect of increasing $\theta_{f}$ follows an inverted $\mathrm{U}$ pattern where the Gini increases at first and then declines after a certain threshold is surpassed. Thus passive PA is the dominant force initially for lower values of $\theta_{f}$. This is followed by reduced inequality as the network nears saturation (i.e., full connectivity) when $\theta_{f}$ is comparatively large and active PA dominates. When some ties are transient however, equalization through the active mechanism begins to take full effect and higher $\theta_{f}$ 's lower inequality across the board.

Our simulation results highlight the phase transition near $\theta_{d}=0$, marking the changing nature of the impact of incumbent tie formation. To shed light on the transition, we run simulations with very small values of $\theta_{d}$ in the neighborhood of $\theta_{d} \approx 0$. Figure $6 \mathrm{~b}$ shows that for sufficiently low, nonzero rates of tie decay within the range $0<\theta_{d} \leq 0.01$ (panels 2 to 5), the relationship between $\theta_{f}$ and inequality roughly follows a U-shaped curve. In this regime, an increase in $\theta_{f}$ decreases inequality to a certain minimum, after which a further increase in $\theta_{f}$ does the opposite and actually increases inequality. As $\theta_{d}$ approaches 0 , this pattern gradually reverts to the shape that characterizes that for $\theta_{d}=0$. The relationship between tie formation and inequality is thus highly nonlinear depending on the tie decay parameter. The implication is that when ties are relatively permanent, any policy aimed to promote connectivity could inadvertently privilege the connected even more.

In addition to overall inequality captured by the Gini coefficient, we also examine the gap between higher and lower human capital agents to shed light on how differential 

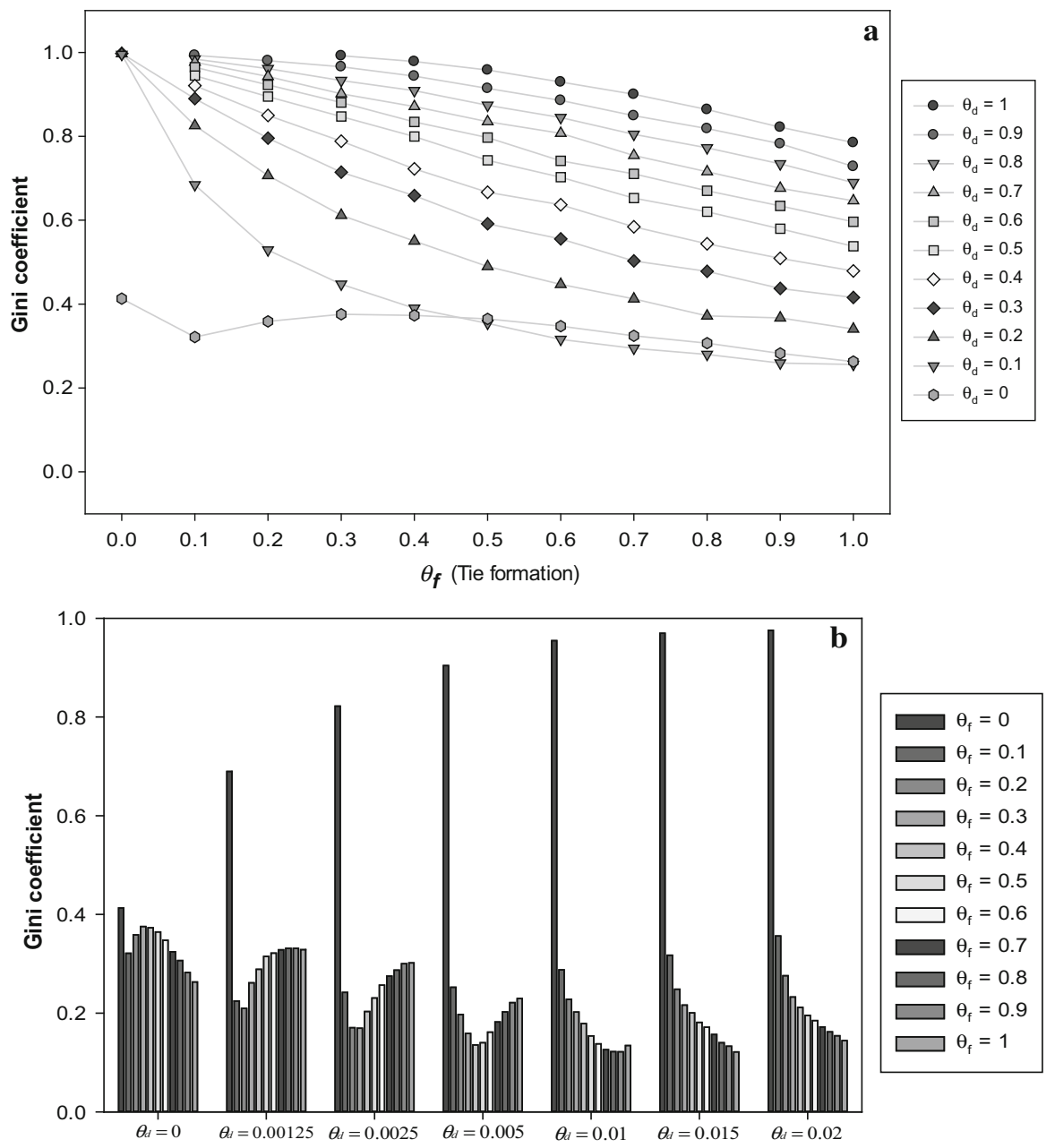

Fig. 6 Relationships between tie formation $\left(\theta_{f}\right)$, decay $\left(\theta_{d}\right)$, and the Gini coefficient. For $\mathbf{b}$, bars for each $\theta_{d}$ panel are ordered from the left in increasing levels of $\theta_{f}$. Values are the mean over five simulation runs each with a different random seed for every parameter configuration

access to human capital affects the trajectories for social capital. ${ }^{12}$ Figure 7 depicts the evolution of agent inequality based on human capital levels for four different parameter settings, each embodying distinct trajectories. Here, the difference in social capital between high (75th percentile or above) and low (25th percentile or below) human capital agents is measured relative to the latter. ${ }^{13}$ Comparing the case for $\theta_{f}=\theta_{d}=0$

\footnotetext{
12 Dynamic analyses of the Gini coefficient (not included here) suggest that the Gini is relatively stable for all parameter settings across time, after the network has evolved to include a sufficient number of agents for reliable Gini calculation.

13 Specifically, inequality is measured at each time point as the difference in average social capital between high (75th percentile and above) and low (25th percentile and below) human capital agents, divided by the average social capital of the low human capital agents.
} 


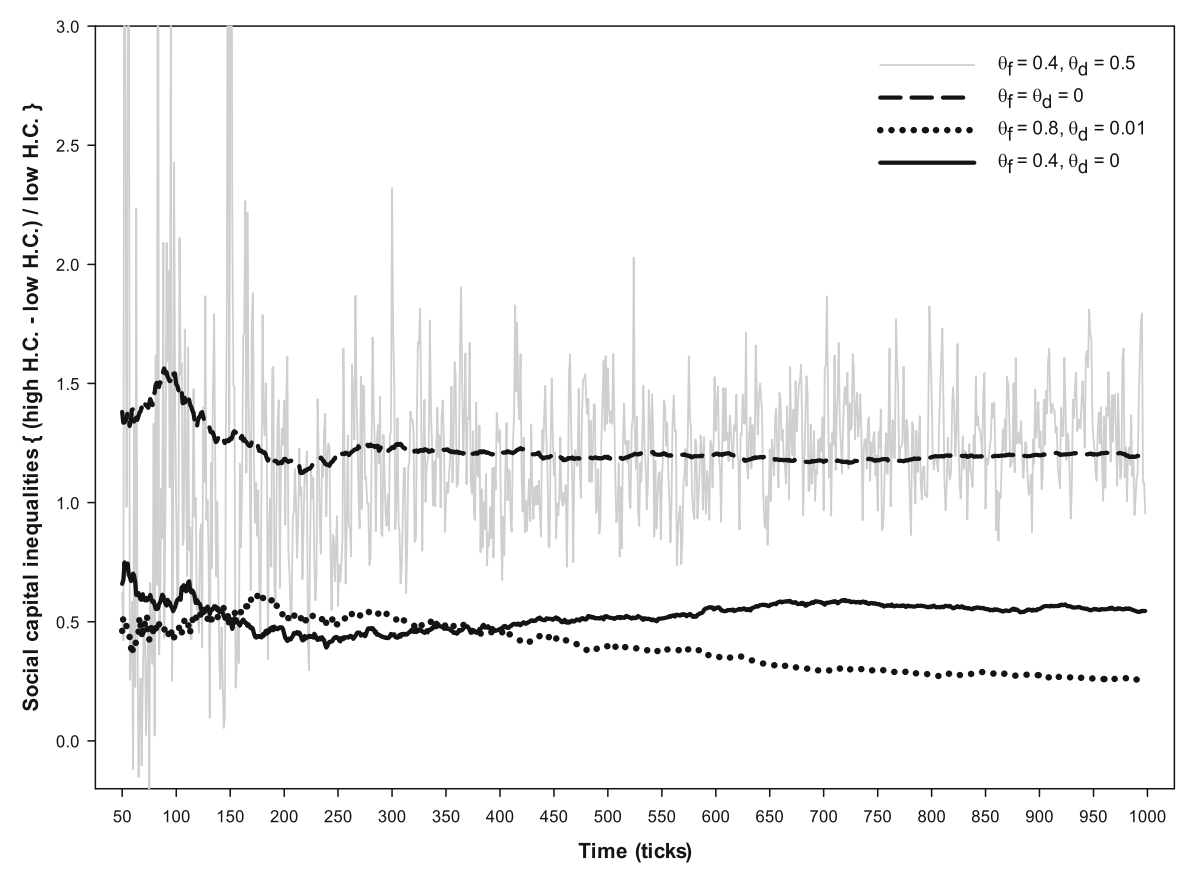

Fig. 7 Differences in social capital between high (75 percentile and above) and low (25 percentile and below) human capital agents across time, calculated by dividing the raw difference in average social capital between high and low human capital agents by the average social capital of low human capital agents. The figure omits values for which $t<50$ due to excessive volatility in values for a small set of agents. Values are the mean over five simulation runs each with a different random seed for every parameter configuration

and that for $\theta_{f}=0.4, \theta_{d}=0.5$, it can be seen that the two settings converge to similar values of inequality in the long run, yet the trajectory for the network with churning is much more volatile. Both configurations yield scale-free degree distributions, but our results reveal that power laws also produce a highly skewed distribution of social capital with the higher human capital agents commanding levels of social capital twice that of the lower human capital agents. The oscillations for the churning case $\left(\theta_{f}=0.4, \theta_{d}=\right.$ 0.5 ) are due to the constant rewiring of links, and it is churning that allows lower human capital agents to improve their social standing vis-à-vis the more privileged ones.

\subsection{Aggregate social capital and agent inequality}

The non-monotonic relationship shown in Fig. 8 between distribution (measured by the Gini) and overall connectivity (measured by total link count) merits an explanation. ${ }^{14}$ As discussed in Sect. 5.2, what matters for connectivity is the ratio $\theta_{f} / \theta_{d}$ since aggregate social capital is strictly increasing in $\theta_{f}$ while strictly decreasing in $\theta_{d}$. The trajectory for inequality on the other hand is dependent on both the magnitude of $\theta_{d}$

\footnotetext{
14 We use link count instead of aggregate social capital to make our results more intuitive, recognizing that the two are qualitatively similar in their behavior across parameter values (see "Appendix 1").
} 


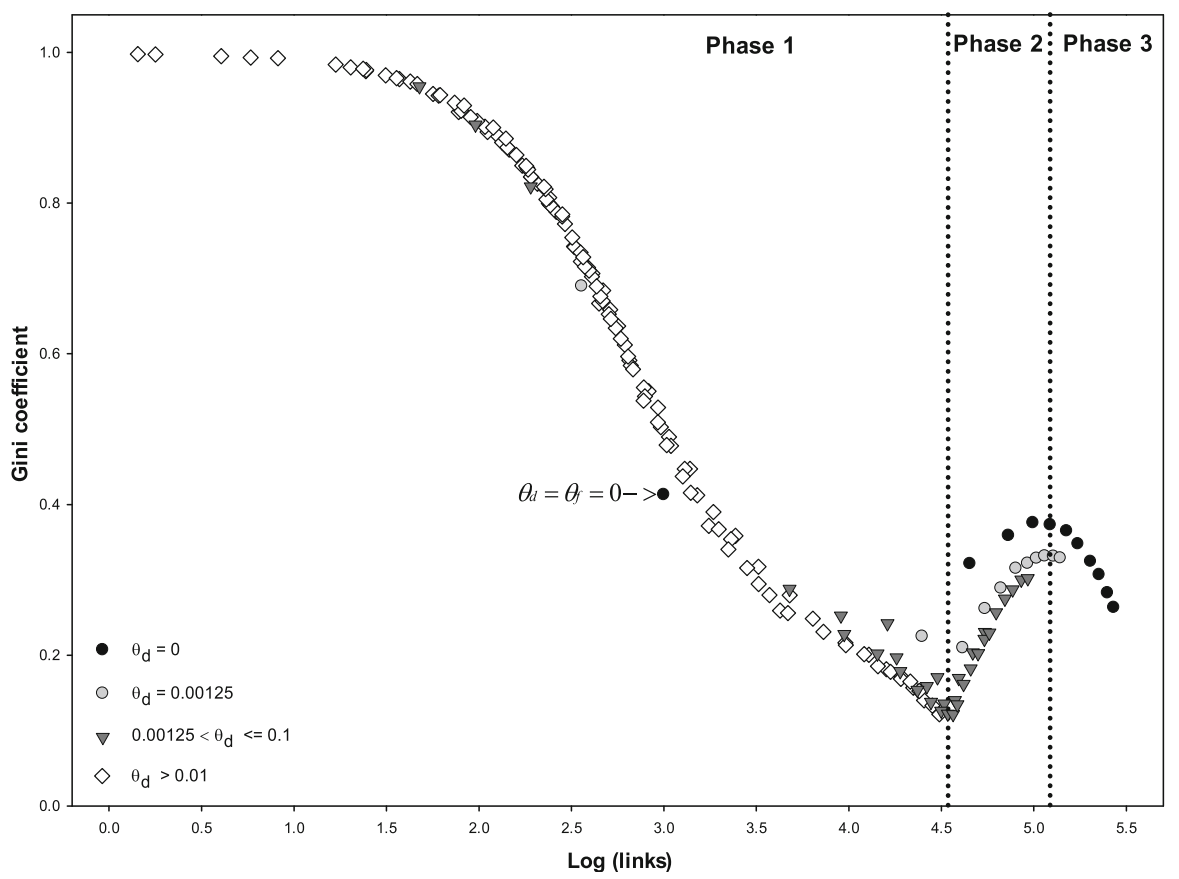

Fig. 8 Relationship between link count and the Gini coefficient. Values are the mean over five simulation runs each with a different random seed for every parameter configuration

and its relative strength. Hence starting from a sparse network, an increase in the ratio $\theta_{f} / \theta_{d}$ generally accomplishes both higher connectivity and a more equal distribution. But if the ratio is increased further by lowering tie decay rate $\theta_{d}$ from a level already close to zero, then a tradeoff ensues where stronger connectivity accompanies greater concentration of social capital. However, a reversal is observed at sufficiently high levels of connectivity with the return of the inverse relationship.

More specifically, there are two transitions occurring at total link count $k \approx 35,000$ and at $k \approx 126,000$, respectively, resulting in three distinct phases. To explain these results recall that an agent acquires social capital through two opposing mechanisms, namely active and passive PA. Which one dominates here hinges on the level of network activity and network density, which in turn depend on the combination of $\theta_{f}$ and $\theta_{d}$. Phase I represents sparse networks with low network densities between 0 and $7 \%$ where the fall in of inequality is driven by three factors. First, low human capital agents maintain a very small number of - if any-links. An increase in $\theta_{f}$ therefore renders their connection gains through active PA comparatively larger. Second, the gains for higher human capital agents being passively linked to are not as large as the gains for lower human capital agents actively initiating connections. Finally, even in sparse networks higher human capital agents command more links and thus have a higher probability for their ties to decay as long as $\theta_{d}>0$. 
Phase II includes networks with moderate density between 7 and $25 \%$. Here inequality increases with aggregate social capital because in moderately dense networks, the gains for lower human capital agents is not as large as in sparse networks for a significant number of high benefit links have already been exploited. This is in contrast to higher human capital agents who benefit from both active and passive attachment mechanisms. Finally, Phase III represents very dense networks nearing link saturation for which inequality falls once again as connectivity increases further. This is due to most incumbents in this regime already having links with most others, and thus a new tie is simply becoming the equalizing vehicle that closes the gap between lower and higher human capital agents.

\subsection{Spatial inequalities}

Space matters in our model as agents' relative positions within the spatial landscape influence the level of social capital that they are able to maintain. We turn now to the locational patterns of agents commanding higher than average social capital, and compare these with those for the rest. Since the amount of aggregate social capital differs across different parameter settings, for comparison purposes we calculate the relative amount of social capital $\overline{\overline{S C}}_{i}$ an agent has as:

$$
\overline{\overline{S C}}_{i}=\frac{S C_{i} / \sum_{i} S C_{i}}{1 / N},
$$

where $N$ is the total number of agents within the network. Thus an agent with a value of $\overline{\overline{S C}}_{i}$ greater than 1 maintains a greater amount of social capital than when mean social capital is uniformly distributed among all agents. We then divide agents into two categories, based on whether they have values of $\overline{\overline{S C}}_{i}$ greater than or less than (or equal to) 1 .

Figure 9 shows the long-run spatial distribution of agents with $\overline{\overline{S C}}_{i}>1$ for select parameter settings, where the nodes are color-coded based on whether they are extroverts or introverts. ${ }^{15}$ Recall that extroverts are unencumbered in connecting with others while introverts are constrained by their spatial reach. ${ }^{16}$ The original PA model with $\theta_{f}=\theta_{d}=0$ is shown in Fig. 9a, where high social capital agents are randomly distributed with a significant number with $\overline{\overline{S C}}_{i}$ greater than 1 on the outer edges of the grid. By contrast, Fig. 9b-d indicates that a higher number of links $(K)$ accompanies the clustering of the high social capital agents toward the center, which in turn implies the lower social capital agents primarily occupying the fringe. Figure $4 \mathrm{e}, \mathrm{f}$ shows that high social capital agents again disperse spatially for even larger values of $K$. What is striking is that the spatial distribution of high social capital agents closest to the center (Fig. 9d) occurs precisely at the parameter setting for which the Gini coefficient is the

\footnotetext{
15 While not shown, due to the random spatial distribution of all agents the blank areas in Fig. 9 are occupied by low social capital agents.

16 Analysis of the spatial distribution of agents based on human capital levels (not shown here) suggests that the spatial distribution of social capital is independent from that of human capital, with no clear emergent pattern.
} 

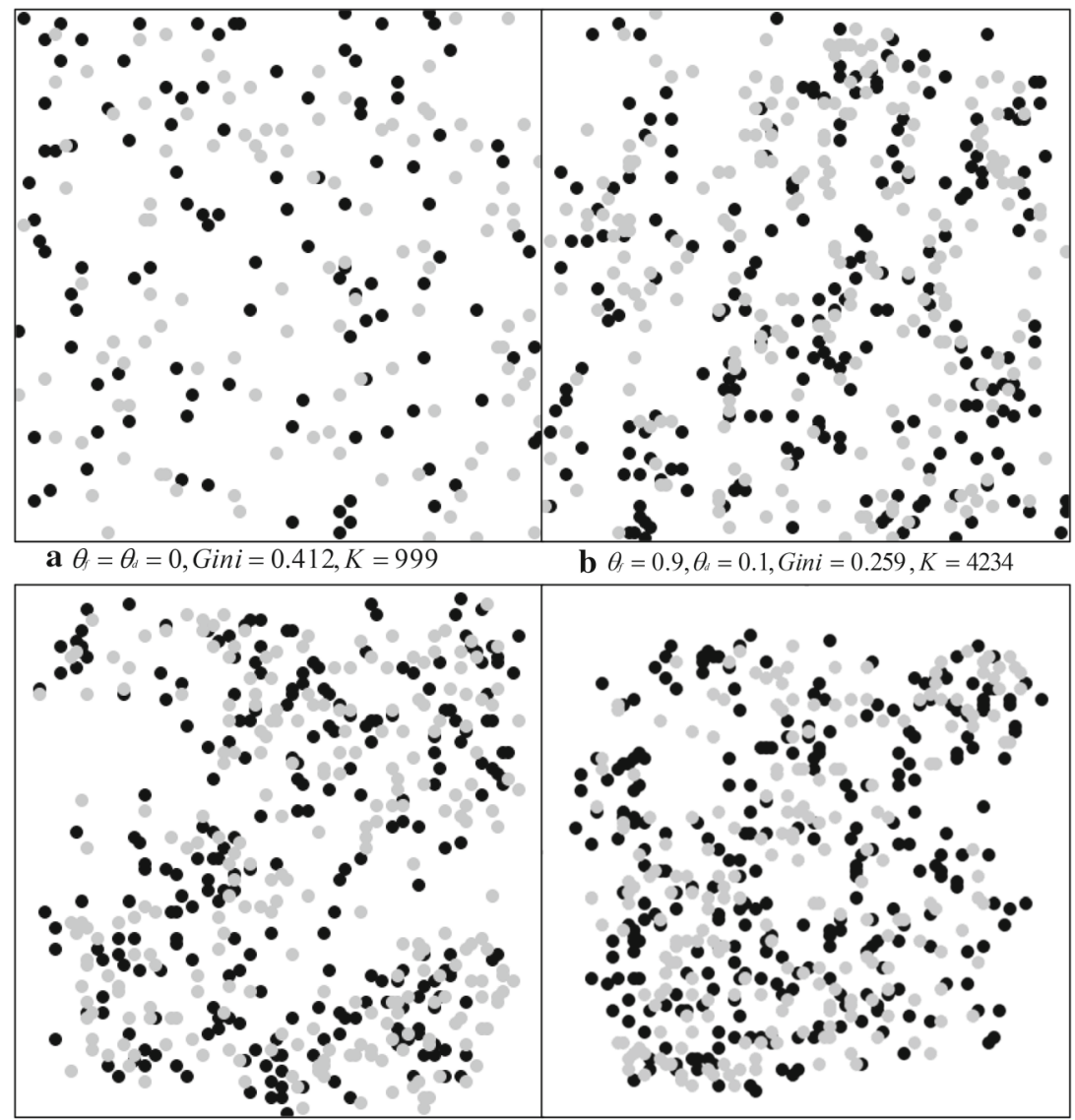

c $\theta_{f}=0.7, \theta_{d}=0.015$, Gini $=0.15, K=22383$ d $\theta_{f}=0.8, \theta_{d}=0.01$, Gini $=0.103, K=35123$

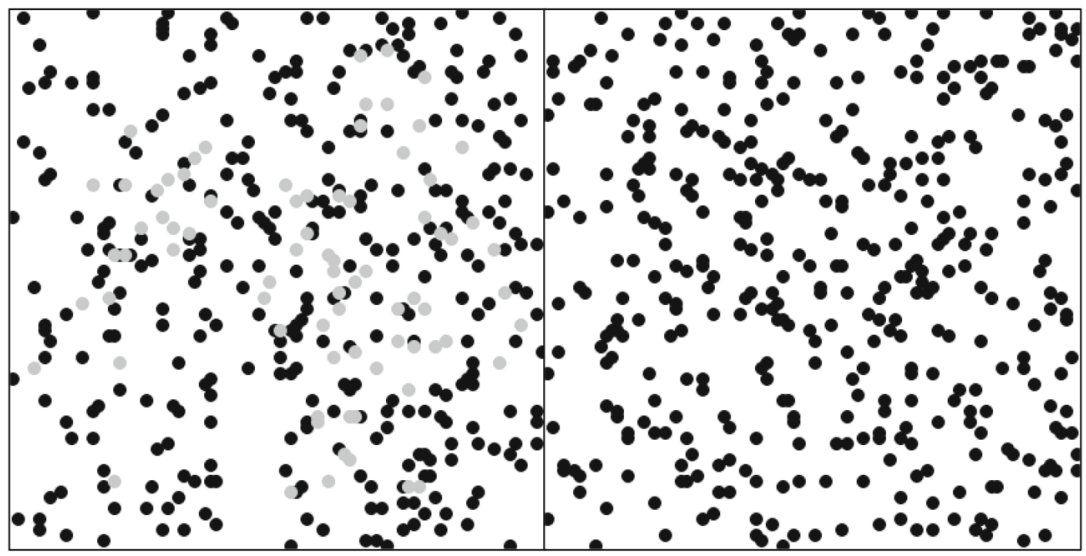

e $\theta_{i}=0.3, \theta_{i}=0$, Gini $=0.367, K=101317 \quad$ f $\theta_{f}=1, \theta_{d}=0$, Gini $=0.263, K=270756$

Fig. 9 Spatial distribution of agents with $\overline{\overline{S C}}_{i}>1$ for representative parameter settings. Black and gray nodes are extroverts and introverts, respectively 
lowest (see Fig. 6b). This implies that, overall, spatial inequality and social capital inequality are inversely related. Other parameter settings with higher Gini coefficients indeed produce a lesser concentration toward the center.

Our results can be explained when we take into consideration the distinct trajectories of link formation as $K$ increases. For sparse networks, the paucity of links lowers the probability of linking to agents further away, since the model prioritizes agents that are close by. This results in agents occupying locally central positions being able to maintain higher levels of social capital, resulting in a more even distribution of such agents within the spatial grid. As density increases, long-distance ties increasingly become likely and as a result agents commanding globally central positions have higher chances of connecting to or being connected. Centrally positioned agents then end up accumulating more social capital than agents on the fringe who have fewer options. However, as density increases even further, the network becomes saturated to the point where central agents lose their advantage, for agents on the fringe are also able to maintain links with distant nodes.

Another key observation is the distinct pattern in the composition of high social capital agents relative to their breed as the number of links increases. In Fig. 9a-d, the distribution of extroverts versus introverts is relatively even suggesting that in lowdensity networks, introverts ceteris paribus have equal opportunities to acquire high levels of social capital. However, Fig. 9e, f reveals that as the number of links crosses a certain threshold, introverts rapidly vanish from the set of high social capital agents. Figure 10a reveals that indeed there is a sharp increase in the absolute difference in average social capital between extroverts and introverts near $\log 4.55 \approx 35,000$, which is precisely the point at which social capital inequality enters the first phase transition (see Fig. 8). While not shown here, this sharp increase in social capital inequality between extroverts and introverts persists even after controlling for the overall higher levels of aggregate social capital in denser networks.

This suggests that the transition from phase 1 to 2 in Fig. 8 not only entails a sharp increase in aggregate inequality, but high levels of inequality between extroverts and introverts as well. At a link count of roughly 100,000, extroverts command levels of social capital more than twice that of the introverts, which is substantial considering that for lower values of $K$ they were virtually identical.

It turns out that disparities increase in network density because of the limited visibility reach $(v)$ of introverts. In the beginning, both extroverts and introverts mainly connect with those close by since pairs that are in the vicinity have a higher chance to form connections. As the network crosses a certain density threshold however, both types exhaust their local possibilities, yet extroverts continue to connect with other agents farther away while introverts are no longer able to establish new ties due to their spatial constraints.

Overall, the tradeoff between spatial and individual inequality suggests that policies geared toward reducing one could adversely affect the other. In addition, our model offers one plausible explanation for the existence of agglomeration economies within a social interactions setting. The essential sources of agglomeration are off-market knowledge exchanges, input and output linkages, as well as labor market pooling (Marshall 1920), which are all driven to some extent by social interactions (Ioannides 2013). Our model suggests that spatial agglomeration benefits individuals in the core 


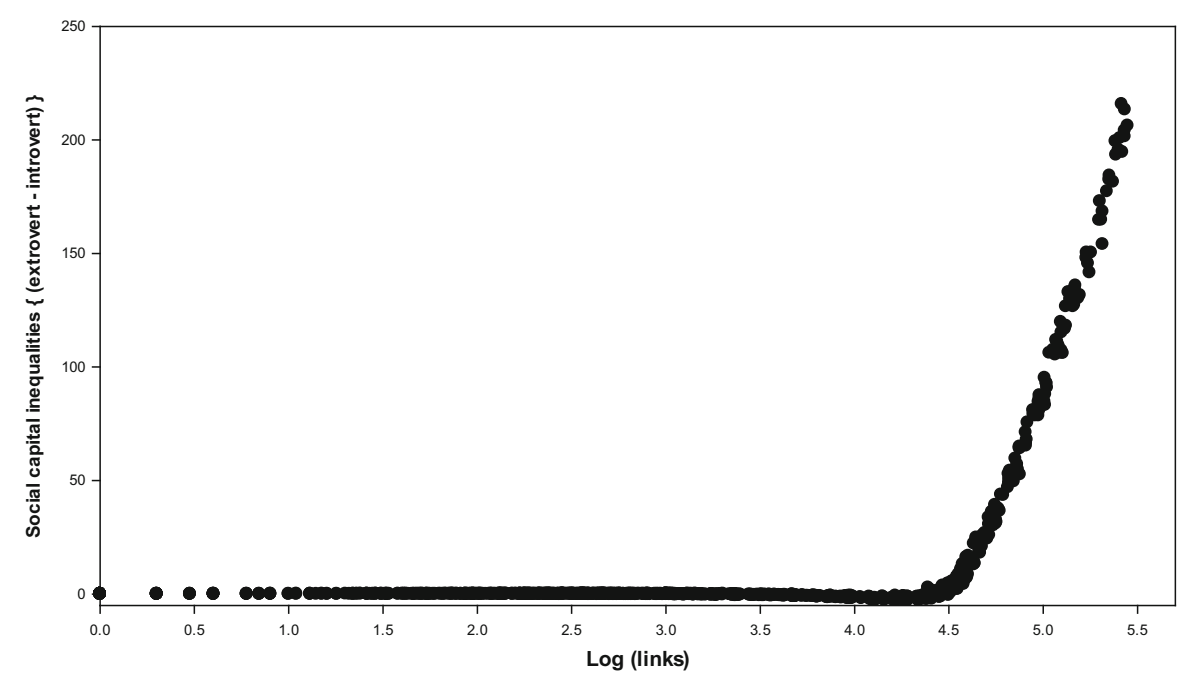

Fig. 10 Differences in social capital between introverts and extroverts as a function of link count, measured as raw differences (extrovert s.c.-introvert s.c.). Values are the mean over five simulation runs each with a different random seed for every parameter configuration

by allowing them to maintain more social connections than those in the periphery, which in turn may result in better economic outcomes.

\section{Conclusions}

We have examined the evolution of degree distributions under different parameter configurations to establish conditions in which the power law is sustained and the cases in which it breaks down. While our presentation has focused on a few select parameter settings, sensitivity analysis reveals that our results are robust across different configurations for world size $L$ and introvert visibility $v$ (see "Appendix 3"). Generally, we find that networks in which ties are scarce and the rate of tie dissolution is relatively high exhibit power law degree distributions. We also find that networks with link dissolution are fundamentally different from those in which ties are relatively permanent, underscoring the importance of distinguishing between the two types of networks. As a classic example, Watts (1999) shows that many social networks with tie decay are characterized by "small-world" properties, and these result in a very different degree distribution from that of networks where ties are permanent, which Barabási and Albert (1999) find to exhibit the power law. In fact, our results suggest that the power law distributions found for networks grown under PA are just a special case of a broader class of networks that take into consideration churning dynamics.

Of particular interest is the concentration of social resources, which we find to be closely related to network density as it evolves in three distinct phases. Sparse networks exhibit a decrease in social capital inequality as network density increases, moderately dense networks exhibit increases in inequality with higher density, and very dense networks exhibit a decrease in inequality as the network reaches full saturation. In a complete (i.e., fully connected) network, no inequality would exist and the level 
of aggregate social capital would be maximal. However, such networks are extremely rare in real life. Our model suggests that due consideration for the relative strength of tie formation and dissolution is warranted when aiming to mitigate disparities over control of network resources. For example, when considering the spread of tacit information, encouraging more networking activity in an ethnic enclave where ties are relatively permanent and dense would have very different results than encouraging such activity among trade association members where ties are weaker and more transient. This relationship between network density and inequality among agents is further complicated when considering the spatial aspects of inequality. We find that spatial inequality is greater - in the form of higher social capital agents being distributed near the core-when inequality among agents overall is low. Our results suggest that we should acknowledge the potential tradeoff between spatial inequality and individual inequality with respect to social resources.

In this paper, we have conceptualized social capital as a network resource rather than an individual resource that an agent is endowed with. This view is motivated by distributional considerations. The poor in particular-due to resource deprivationare more likely to utilize membership in community networks that exchange help in crises than to resort to individual coping mechanisms (Gans 1962). Our results highlight counterintuitively that simply encouraging more networking activity among individuals may not result in the disadvantaged benefiting from the increased intensity of social interactions.

Although this study does not pursue it, the current specification has enabled a new kind of analysis for furthering the understanding of network dynamics. Our model establishes the impact of connectivity on social capital through Eq. (6). The accumulation of social capital, however, likely results in higher betweenness centrality (Freeman 1977), which directly triggers additional rounds of tie decay and formation. An examination of closed-loop feedback effects of this type is beyond the scope of the present study but should be attempted in the future as an extension.

Finally, while social capital has mainly been studied within the context of social networks, we note that it can be generalized to other domains-such as economic networks - in which resources obtained from network ties are of central importance. Our model's simple definition of social capital to be the human capital sum of link neighbors allows our framework to be readily extended to other types of networks, possibly by substituting human capital with a different resource of importance. We hope that our demonstration highlighting the complexity of network behavior in space and in the presence of churning dynamics will stimulate further investigation of the implications for differential spatial patterns and social inequality.

Open Access This article is distributed under the terms of the Creative Commons Attribution 4.0 International License (http://creativecommons.org/licenses/by/4.0/), which permits unrestricted use, distribution, and reproduction in any medium, provided you give appropriate credit to the original author(s) and the source, provide a link to the Creative Commons license, and indicate if changes were made. 


\section{Appendix 1}

See Table 1.

Table 1 Relationship between $\theta_{f}, \theta_{d}$, and (a) aggregate social capital $\sum_{i} S C_{i}$ (b) link count $(k)$

\begin{tabular}{|c|c|c|c|c|c|c|c|c|c|c|c|}
\hline \multirow[t]{2}{*}{$\theta_{f}$} & \multicolumn{11}{|c|}{$\theta_{d}$} \\
\hline & 0 & 0.1 & 0.2 & 0.3 & 0.4 & 0.5 & 0.6 & 0.7 & 0.8 & 0.9 & 1 \\
\hline
\end{tabular}

(a) Aggregate social capital $\left(\sum_{i} S C_{i}\right)$

$\begin{array}{llllllllllll}0 & 1215.4 & 5.0 & 2.1 & 1.8 & 0.6 & -{ }^{\mathrm{a}} & - & - & - & - & - \\ 0.1 & 49,813.1 & 578.3 & 251.0 & 150.7 & 93.8 & 67.6 & 42.7 & 29.8 & 20.2 & 8.2 & - \\ 0.2 & 80,618.1 & 1106.5 & 508.4 & 306.1 & 208.1 & 137.0 & 95.7 & 71.5 & 49.3 & 22.4 & 1.7 \\ 0.3 & 109,908.4 & 1649.8 & 734.5 & 474.6 & 326.3 & 223.3 & 163.9 & 125.6 & 90.3 & 42.6 & 9.9 \\ 0.4 & 136,943.3 & 2199.3 & 990.4 & 618.1 & 435.0 & 288.0 & 227.0 & 173.6 & 115.8 & 71.8 & 26.8 \\ 0.5 & 168,187.3 & 2703.4 & 1267.0 & 780.0 & 542.6 & 390.3 & 295.5 & 230.9 & 169.7 & 105.9 & 55.6 \\ 0.6 & 189,633.4 & 3280.7 & 1513.8 & 915.7 & 680.7 & 492.3 & 394.0 & 288.7 & 219.6 & 166.8 & 99.6 \\ 0.7 & 219,314.2 & 3821.6 & 1783.3 & 1137.7 & 795.3 & 603.4 & 480.3 & 380.8 & 274.0 & 214.3 & 135.2 \\ 0.8 & 242,426.7 & 4307.1 & 2044.5 & 1279.0 & 932.3 & 706.1 & 550.8 & 445.6 & 349.5 & 265.1 & 185.7 \\ 0.9 & 267,520.2 & 4883.1 & 2309.4 & 1485.6 & 1094.1 & 808.6 & 648.7 & 541.8 & 420.6 & 331.5 & 262.7 \\ 1 & 285,562.4 & 5348.8 & 2547.3 & 1631.3 & 1227.8 & 921.6 & 763.8 & 601.1 & 517.2 & 434.4 & 334.1 \\ \Delta_{\text {SC }} & 57.6 & 1.1 & 0.6 & 0.4 & 0.4 & 0.3 & 0.3 & 0.3 & 0.3 & 0.5 & -\end{array}$

(b) Link count $(K)$

\begin{tabular}{llllllllllll}
0 & 999 & 4 & 2 & 2 & 1 & - & - & - & - & - & - \\
0.1 & 45,244 & 469 & 207 & 126 & 78 & 57 & 38 & 25 & 17 & 7 & - \\
0.2 & 73,048 & 930 & 412 & 249 & 172 & 112 & 81 & 61 & 43 & 21 & 1 \\
0.3 & 99,041 & 1384 & 615 & 389 & 263 & 183 & 133 & 109 & 74 & 37 & 8 \\
0.4 & 122,936 & 1853 & 827 & 512 & 356 & 239 & 192 & 146 & 98 & 63 & 24 \\
0.5 & 150,770 & 2305 & 1065 & 652 & 446 & 326 & 243 & 191 & 143 & 90 & 47 \\
0.6 & 173,607 & 2815 & 1296 & 780 & 565 & 409 & 329 & 240 & 185 & 140 & 83 \\
0.7 & 202,849 & 3252 & 1514 & 961 & 662 & 505 & 394 & 319 & 232 & 180 & 120 \\
0.8 & 226,062 & 3718 & 1748 & 1092 & 790 & 586 & 467 & 374 & 292 & 229 & 160 \\
0.9 & 250,995 & 4251 & 1978 & 1264 & 927 & 681 & 549 & 453 & 355 & 282 & 224 \\
1 & 270,693 & 4683 & 2232 & 1398 & 1036 & 776 & 640 & 517 & 434 & 366 & 283 \\
$\Delta_{K}$ & 62.0 & 1.2 & 0.6 & 0.4 & 0.4 & 0.3 & 0.3 & 0.3 & 0.3 & 0.5 & - \\
\hline
\end{tabular}

$\Delta$ is the average change (in multiples) in (a) social capital and (b) link count compared to the next highest $\theta_{d}$ value. $N=1000, L=100, v=15$. Values are averaged over 5 simulations

${ }^{\text {a }}$ For lower values of $\theta_{f}$, sufficiently high values of $\theta_{d}$ prohibit the network from maintaining any links, resulting in no social capital

\section{Appendix 2}

See Table 2. 
Table 2 Relationship between $\theta_{f}, \theta_{d}$, and the Gini coefficient

\begin{tabular}{|c|c|c|c|c|c|c|c|c|c|}
\hline \multirow[t]{2}{*}{$\theta_{f}$} & \multicolumn{9}{|l|}{$\theta_{d}$} \\
\hline & 0 & 0.00125 & 0.0025 & 0.005 & 0.01 & 0.015 & 0.02 & 0.1 & 0.2 \\
\hline 0 & 0.413 & 0.690 & 0.822 & 0.904 & 0.955 & 0.969 & 0.976 & 0.995 & 0.998 \\
\hline 0.1 & 0.321 & 0.225 & 0.242 & 0.252 & 0.288 & 0.318 & 0.356 & 0.684 & 0.825 \\
\hline 0.2 & 0.358 & 0.210 & 0.170 & 0.197 & 0.228 & 0.249 & 0.276 & 0.529 & 0.707 \\
\hline 0.3 & 0.376 & 0.261 & 0.170 & 0.159 & 0.202 & 0.217 & 0.233 & 0.447 & 0.612 \\
\hline 0.4 & 0.373 & 0.289 & 0.204 & 0.135 & 0.179 & 0.201 & 0.211 & 0.390 & 0.550 \\
\hline 0.5 & 0.365 & 0.315 & 0.231 & 0.140 & 0.154 & 0.181 & 0.195 & 0.354 & 0.490 \\
\hline 0.6 & 0.347 & 0.322 & 0.257 & 0.162 & 0.138 & 0.171 & 0.185 & 0.316 & 0.447 \\
\hline 0.7 & 0.324 & 0.329 & 0.275 & 0.182 & 0.126 & 0.157 & 0.172 & 0.295 & 0.412 \\
\hline 0.8 & 0.307 & 0.331 & 0.287 & 0.203 & 0.122 & 0.140 & 0.162 & 0.280 & 0.372 \\
\hline 0.9 & 0.282 & 0.331 & 0.300 & 0.221 & 0.122 & 0.133 & 0.154 & 0.259 & 0.367 \\
\hline 1 & 0.263 & 0.329 & 0.302 & 0.230 & 0.134 & 0.122 & 0.144 & 0.256 & 0.340 \\
\hline$\Delta_{\text {Gini }}$ & 0.027 & 0.114 & 0.170 & 0.052 & -0.073 & -0.068 & -0.362 & -0.215 & -0.130 \\
\hline \multirow[t]{2}{*}{$\theta_{f}$} & \multicolumn{9}{|l|}{$\theta_{d}$} \\
\hline & 0.3 & 0.4 & 0.5 & 0.6 & 0.7 & 0.8 & 0.9 & 1 & \\
\hline 0 & 0.998 & 0.798 & - & - & - & - & - & - & \\
\hline 0.1 & 0.890 & 0.921 & 0.945 & 0.964 & 0.977 & 0.984 & 0.993 & - & \\
\hline 0.2 & 0.795 & 0.849 & 0.894 & 0.922 & 0.943 & 0.962 & 0.980 & 0.398 & \\
\hline 0.3 & 0.715 & 0.789 & 0.847 & 0.881 & 0.901 & 0.933 & 0.966 & 0.992 & \\
\hline 0.4 & 0.658 & 0.723 & 0.799 & 0.834 & 0.871 & 0.909 & 0.944 & 0.978 & \\
\hline 0.5 & 0.591 & 0.667 & 0.742 & 0.797 & 0.835 & 0.874 & 0.914 & 0.958 & \\
\hline 0.6 & 0.555 & 0.637 & 0.703 & 0.741 & 0.807 & 0.845 & 0.886 & 0.929 & \\
\hline 0.7 & 0.503 & 0.584 & 0.653 & 0.710 & 0.754 & 0.805 & 0.849 & 0.900 & \\
\hline 0.8 & 0.478 & 0.543 & 0.620 & 0.670 & 0.716 & 0.772 & 0.819 & 0.864 & \\
\hline 0.9 & 0.437 & 0.509 & 0.579 & 0.634 & 0.676 & 0.735 & 0.782 & 0.822 & \\
\hline 1 & 0.416 & 0.479 & 0.538 & 0.596 & 0.646 & 0.690 & 0.728 & 0.785 & \\
\hline$\Delta_{\text {Gini }}$ & -0.062 & -0.085 & -0.056 & -0.046 & -0.045 & -0.040 & 0.032 & - & \\
\hline
\end{tabular}

$\Delta_{\text {Gini }}$ is the average change in the Gini coefficient compared to the next highest $\theta_{d}$ value. $N=1000$, $L=100, v=15$. Values are averaged over 5 simulations

\section{Appendix 3}

See Fig. 11. 

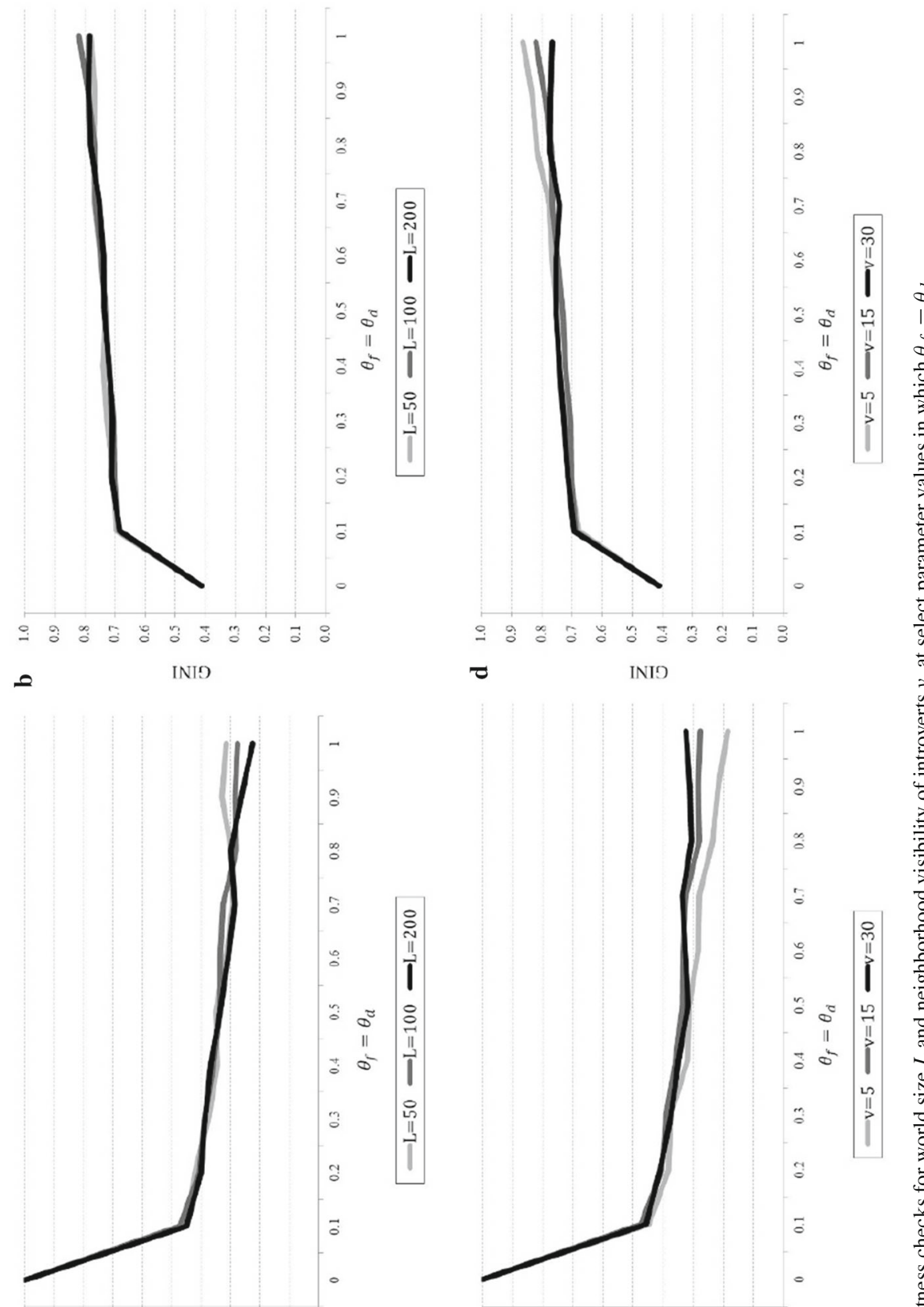

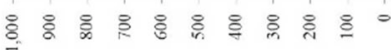

๘ (y) LNกOJ Y YNIT
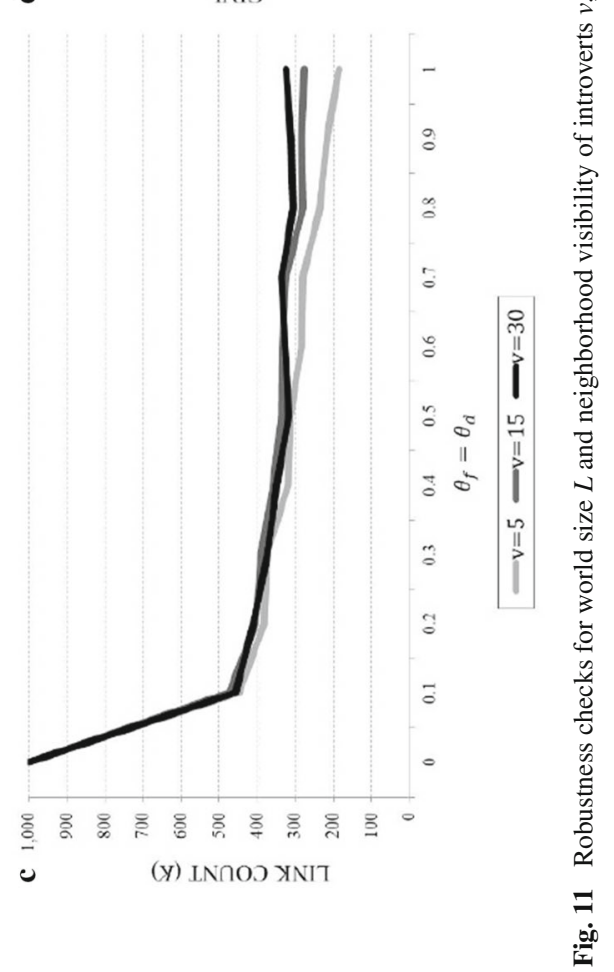


\section{Appendix 4}

\section{Details of the model according to the ODD protocol}

\section{Design concepts}

- Basic principles: see Sect. 3.

- Emergence: We look for the emergence of a power law distribution under different specifications of the tie formation and dissolution parameters, as well as under what circumstances the power law breaks down. We also look for the emergence of high and low inequalities in social capital among agents as well as spatial patterns of inequality based on different parameter settings.

- Adaptation: There is no explicit adaptation of the agents in terms of changes in static state variables. Nonetheless, the agents adapt their behavior with respect to their levels of social capital, with higher social capital agents deleting more ties due to the limited social effort they can exert.

- Objective: The agents' objective is to maximize their social capital in each time frame, by connecting to another agent that preferentially has a higher level of human capital (which would imply that these agents also have higher connectivity). However, the agents are constrained by distance, being able to connect more easily to others who are closer to themselves. Introverts are also constrained by their neighborhood visibility, only being able to connect to others within a fixed threshold distance.

- Sensing: Agents use probabilities in deciding which new connection to form and which tie to dissolve. The probabilities are calculated based on the information set that reveals the human capital and connectivity levels of other agents, as well as their relative distance to these agents. Introverts are assumed to be able to sense this information only for agents within their visibility reach, while extroverts are assumed to be fully knowledgeable of all information regarding all agents in the network.

- Interaction: Agents interact directly with each other through the formation and the dissolution of links, which in the real world would represent the re-evaluation of social connections. Introverts interact only with other agents within their visibility reach, while extroverts interact with all other agents. All agents interact with all possible others in each time frame.

- Stochasticity: Stochastic processes are used to assign the spatial position of each agent at birth. In addition, the human capital of each agent is randomly drawn from a uniform distribution within the interval $[0,1]$. Stochasticity also appears in all tie formation and dissolution processes, for these processes are determined based on probabilities which may yield different results based on different random number seeds.

- Collectives: The collective-or aggregate-level of social capital, the degree distribution, average levels of social capital for extroverts versus introverts, as well as spatial patterns of social capital inequality are represented in the model, and emerge from the behavioral characteristics of agents. 
- Observation: We track the evolution of degree distribution, individual and aggregate levels of social capital, and inequality in social capital among agents as well as spatially. We use numeric data representations as well as graphs to present these outputs of interest, and also utilize maps of agents to present spatial patterns.

\section{Initialization}

At the beginning of every simulation $(t=0)$, two agents linked to each other are placed uniformly and randomly on a respective patch. These two agents are assigned into the introvert or extrovert breed randomly with equal probability, and their human capital is drawn from a uniform distribution with $[0,1]$ support. Introverts are assigned a specific neighborhood visibility reach value. In addition, the tie formation and dissolution parameters are set globally at specific values, and these values are assumed to be constant across agents.

\section{Input data}

The environment is assumed to be generic, and thus the model has no input data.

\section{Submodels}

See the process overview and scheduling section for details on each procedure.

\section{References}

Adamic LA, Huberman BA (2000) Power-law distribution of the world wide web. Science 287(5461):2115 Aral S, Muchnik L, Sundararajan A (2009) Distinguishing influence-based contagion from homophilydriven diffusion in dynamic networks. Proc Natl Acad Sci 106(51):21544-21549

Barabasi A-L (2002) Linked: how everything is connected to everything else and what it means for business, science, and everyday life. Plume, New York

Barabási A-L, Albert R (1999) Emergence of scaling in random networks. Science 286(5439):509-512

Batty M (2013) The new science of cities. MIT Press, Cambridge

Bianconi G, Barabási A-L (2001) Competition and multiscaling in evolving networks. Europhys Lett 54(4):436

Borgatti SP, Cross R (2003) A relational view of information seeking and learning in social networks. Manag Sci 49(4):432-445

Browning CR, Dietz RD, Feinberg SL (2004) The paradox of social organization: networks, collective efficacy, and violent crime in urban neighborhoods. Soc Forces 83(2):503-534

Burt RS (1992) Structural holes: the social structure of competition. Harvard University Press, Cambridge Burt RS (2005) Brokerage and closure: an introduction to social capital. Oxford University Press, Oxford

Campbell KE (1990) Networks past: a 1939 Bloomington neighborhood. Soc Forces 69(1):139-155

Cassi L, Plunket A (2014) Proximity, network formation and inventive performance: in search of the proximity paradox. Ann Reg Sci 53(2):395-422

Castellano C, Marsili M, Vespignani A (2000) Nonequilibrium phase transition in a model for social influence. Phys Rev Lett 85(16):3536

Coleman JS (1988) Social capital in the creation of human capital. Am J Sociol 94:S95-S120

Cornwell B, Dokshin FA (2014) The power of integration: affiliation and cohesion in a diverse elite network. Soc Forces. doi:10.1093/sf/sou068 
Cuperman R, Ickes W (2009) Big five predictors of behavior and perceptions in initial dyadic interactions: personality similarity helps extraverts and introverts, but hurts "disagreeables". J Pers Soc Psychol 97(4):667

De Montis A, Chessa A, Campagna M, Caschili S, Deplano G (2009) Complex networks analysis of commuting. In: Reggiani A, Nijkamp P (eds) Complexity and spatial networks. Springer, Berlin, Heidelberg pp 239-255

de Solla Price DJ (1965) Networks of scientific papers. Science 149(3683):510-515. doi:10.1126/science. 149.3683.510

Erdos P, Rényi A (1960) On the evolution of random graphs. Publ Math Inst Hung Acad Sci 5:17-61

Freeman L (1977) A set of measures of centrality based on betweenness. Sociometry 40(1):35-41

Fritsch M, Kauffeld-Monz M (2010) The impact of network structure on knowledge transfer: an application of social network analysis in the context of regional innovation networks. Ann Reg Sci 44(1):21-38

Gans HJ (1962) The urban villagers: group and class in the life of Italians-Americans. Free Press of Glencoe, New York

Granovetter M (1973) The strength of weak ties. Am J Sociol 78(6):1360-1380

Granovetter M (2005) The impact of social structure on economic outcomes. J Econ Perspect 19(1):33-50

Greve A, Salaff JW (2003) Social networks and entrepreneurship. Entrep Theory Pract 28(1):1-22

Hampton K, Sessions L, Her E, Rainie L (2009) Social isolation and new technology. Pew Internet \& American Life Project, Washington

Holme P, Newman ME (2006) Nonequilibrium phase transition in the coevolution of networks and opinions. Phys Rev E 74(5):056108

Ioannides YM (2013) From neighborhoods to nations: the economics of social interactions. Princeton University Press, Princeton

Ioannides YM, Topa G (2010) Neighborhood effects: accomplishments and looking beyond them. J Reg Sci 50(1):343-362

Jackson MO (2008) Social and economic networks, vol 3. Princeton University Press, Princeton

Kalmijn M (1998) Intermarriage and homogamy: causes, patterns, trends. Annu Rev Sociol 24:395-421

Karnstedt M, Hennessy T, Chan J, Basuchowdhuri P, Hayes C, Strufe T (2010) Churn in social networks. In: Furht B (ed) Handbook of social network technologies and applications. Springer, US, pp 185-220

Koka BR, Madhavan R, Prescott JE (2006) The evolution of interfirm networks: environmental effects on patterns of network change. Acad Manag Rev 31(3):721-737

Kosmidis K, Havlin S, Bunde A (2008) Structural properties of spatially embedded networks. Europhys Lett 82(4):48005

Lin N (2001) Social capital: a theory of social structure and action. Cambridge University Press, Cambridge

Lin N, Dumin M (1986) Access to occupations through social ties. Soc Netw 8(4):365-385

Madden M (2012) Privacy management on social media sites. Technical report, Pew Internet and American Life Project. Retrieved from http://www.pewinternet.org/ /media//Files/Reports/2012/PIP_Privacy_ management_on_social_media_sites_022412.pdf

Mansury Y, Gulyás L (2007) The emergence of Zipf's Law in a system of cities: an agent-based simulation approach. J Econ Dyn Control 31(7):2438-2460

Mansury Y, Shin J (2015) Size, connectivity, and tipping in spatial networks: theory and empirics. Comput Environ Urban Syst 54:428-437

Marshall A (1920) Principles of economics. MacMillan, London

Mayhew BH, McPherson M, Rotolo T, Smith-Lovin L (1995) Sex and ethnic heterogeneity in face-to-face groups in public places: an ecological perspective on social interaction. Soc Forces 74:15-52

McPherson M, Smith-Lovin L, Cook JM (2001) Birds of a feather: homophily in social networks. Annu Rev Sociol 27:415-444

Mehra A, Kilduff M, Brass DJ (1998) At the margins: a distinctiveness approach to the social identity and social networks of underrepresented groups. Acad Manag J 41(4):441-452

Merton RK (1968) The Matthew effect in science. Science 159(3810):56-63

Miller JH, Page SE (2009) Complex adaptive systems: an introduction to computational models of social life. Princeton University Press, Princeton

Moretti E (2004) Estimating the social return to higher education: evidence from longitudinal and repeated cross-sectional data. J Economet 121(1-2):175-212

Nijkamp P, Rose A, Kourtit K (2014) Regional science matters: studies dedicated to Walter Isard. Springer, New York

Portes A (1998) Social capital: its origins and applications in modern sociology. Annu Rev Sociol 24:25 
Putnam RD (2001) Bowling alone. The collapse and revival of American community. Simon and Schuster, New York

Railsback SF, Grimm V (2011) Agent-based and individual-based modeling: a practical introduction. Princeton University Press, Princeton

Sampson RJ (2004) Networks and neighbourhoods: the implications of connectivity for thinking about crime in the modern city. In: McCarthy H, Miller P, Skidmore P (eds) Who Governs in an Interconnected World? (Chap. 12). Network Logic, Demos, pp 157-166. Retrieved from http://www.demos.co.uk/ files/File/networklogic12sampson.pdf

Sasovova Z, Mehra A, Borgatti SP, Schippers MC (2010) Network churn: the effects of self-monitoring personality on brokerage dynamics. Adm Sci Q 55(4):639-670

Smith A, Duggan M (2013) Online dating \& relationships. Pew Internet \& American Life Project, Washington

Solé RV, Manrubia SC, Luque B, Delgado J, Bascompte J (1996) Phase transitions and complex systems: simple, nonlinear models capture complex systems at the edge of chaos. Complexity 1(4):13-26

Tobler WR (1970) A computer movie simulating urban growth in the detroit region. Econ Geogr 46:234-240

Torrens PM (2007) A geographic automata model of residential mobility. Environ Plan B Plan Des 34(2):200-222

Torrens PM (2010) Agent-based models and the spatial sciences. Geogr Compass 4(5):428-448

Tversky A, Kahneman D (1981) The framing of decisions and the psychology of choice. Science 211(4481):453-458

Vega-Redondo F (2007) Complex social networks. Cambridge University Press, Cambridge

Verbrugge LM (1983) A research note on adult friendship contact: a dyadic perspective. Soc Forces 62:7883

Watts DJ (1999) Small worlds: the dynamics of networks between order and randomness. Princeton University Press, Princeton

Watts DJ, Strogatz SH (1998) Collective dynamics of 'small-world' networks. Nature 393(6684):440-442

Wellman B (1996) Are personal communities local? A Dumptarian reconsideration. Soc Netw 18(4):347354

Wilensky U (1999) NetLogo. Center for connected learning and computer-based modeling. Northwestern University, Evanston

Wilson KL, Portes A (1980) Immigrant enclaves: an analysis of the labor market experiences of Cubans in Miami. Am J Sociol 86:295-319

Xie Y, Batty M, Zhao K (2007) Simulating emergent urban form using agent-based modeling: Desakota in the Suzhou-Wuxian region in China. Ann Assoc Am Geogr 97(3):477-495

Zipf GK (1949) Human behavior and the principle of least effort. Hafner, New York 